\title{
RAMANUJAN'S UNPUBLISHED MANUSCRIPT ON THE PARTITION AND TAU FUNCTIONS WITH PROOFS AND COMMENTARY
}

\author{
Bruce C. Berndt and Ken Ono \\ Dedicated to our good friend George Andrews on his 60th birthday
}

\section{Introduction}

When Ramanujan died in 1920, he left behind an incomplete, unpublished manuscript in two parts on the partition function $p(n)$ and, in contemporary terminology, Ramanujan's tau-function $\tau(n)$. The first part, beginning with the Roman numeral $\mathrm{I}$, is written on 43 pages, with the last nine comprising material for insertion in the foregoing part of the manuscript. G. H. Hardy extracted a portion of Part I providing proofs of Ramanujan's congruences for $p(n)$ modulo 5, 7, and 11 and published it in 1921 [83], [85, pp. 232-238] under Ramanujan's name. In a footnote, Hardy remarks, "The manuscript contains a large number of further results. It is very incomplete, and will require very careful editing before it can be published in full. I have taken from it the three simplest and most striking results, ...." In 1952, J. M. Rushforth [92] published several further results, mostly on $\tau(n)$, from Part I. In 1977, R. A. Rankin [88] discussed several congruences for $\tau(n)$ found in Part I. Part II has not been discussed in the literature. Part I was not made available to the public until 1988 when it was photocopied in its original handwritten form and published with Ramanujan's lost notebook [86]. The existence of Part II was first pointed out by B. J. Birch [26] in 1975, but, like Part I, it also was hidden from the public until 1988, when a handwritten copy made by G. N. Watson was photocopied for [86]. Several theorems and proofs in this manuscript had not previously appeared before 1988 .

The manuscript arises from the last three years of Ramanujan's life. It may have been written in nursing homes and sanitariums in 1917-1919, when we know, from letters that Ramanujan wrote to Hardy during this time [25, pp. 192-193], that Ramanujan was thinking deeply about partitions, or, more likely, it may have been written in India during the last year of his life. According to Rushforth [92], the manuscript was sent to Hardy by "Ramanujan a few months before the latter's death in 1920." If this is true, then it probably was enclosed with Ramanujan's last letter to Hardy, dated January 12, 1920 [25, pp. 220-223]. There is no mention of the manuscript in the extant portion of the letter; part of the letter has been lost. The manuscript was given by Hardy in 1928 to G. N. Watson, who had

The first author thanks the National Security Agency for its generous support.

The second author thanks the National Science Foundation and the National Security Agency for their generous support.

Typeset by $\mathcal{A} \mathcal{M S}-\mathrm{TEX}_{\mathrm{E}}$ 
it in his possession until he died in 1965. At the suggestion of Rankin, Part I was sent shortly thereafter to the library of Trinity College, Cambridge, where it still resides. Watson's copy of Part II can be found in the library of Oxford's Mathematical Institute. We do not know if Ramanujan's orginal copy of Part II exists. For further historical information, see Rankin's two papers [88], [90].

Since many of the proofs in this manuscript had not been published before their appearance in handwritten form with the lost notebook [86], since many details were omitted by Ramanujan, since mathematicians have established results either proved or asserted in the manuscript since it was written, and since the manuscript contains many unproved claims, the purpose of this paper is to present the manuscript in its entirety, offer some additional details, and provide extensive commentary on it. Although many of the results in this manuscript have been proven or explained within a greater context in the works of P. Deligne, J.-P. Serre, H. P. F. SwinnertonDyer, and others, we were delighted to find a number of surprising new gems. For example, Ramanujan's claims (14.1)-(14.6) and many of the assertions in both Sections 15 and 16 were unexpected and entirely new to both authors. Moreover, in proving the claims in Section 14, the second author was led, by the "shape of Ramanujan's claims," to several new general results regarding the distribution of the partition function modulo every prime $m \geq 5$ [73]. Part II, beginning with Section 20, is also fascinating, for it contains Ramanujan's proof, albeit lacking in many details, of his conjectured congruences for $p(n)$ modulo arbitrary integral powers of 5 .

Several editorial decisions needed to be made in our presentation of the manuscript.

1) The nine pages of insertions at the end of Part I were interposed at their intended positions.

2) None of Ramanujan's footnotes, such as "For a direct proof of this see," were completed in the manuscript. We have executed their completions, but we do not claim that they are what Ramanujan had in mind.

3) Due to Ramanujan's failure to tag certain equalities, the manuscript contains incomplete references, such as "... deduce from ( ) and ( ) ...." We have added the tags and inserted the equation numbers. Difficulties arose when tags needed to be inserted at places between already existing tags with consecutive numbers. We appended letters on such tags; e.g., (6.6a) lies between (6.6) and (6.7).

4) As with most of his mathematics, Ramanujan provided very few details in this manuscript. In Part I, Ramanujan indicates, at more than one place, that this is the first of two papers that he intends to write on $p(n)$ and $\tau(n)$. It is clear that as Ramanujan wrote the manuscript he continued to discover more and more theorems on the subject, and so he more and more frequently recorded his results with the promise that he would provide details in his next paper. Thus, details become more sparse as the manuscript progresses, so that in the last third of the manuscript there are hardly any details at all. However, rather than returning in Part II to the details omitted in Part I, Ramanujan sketched his proofs of the congruences for $p(n)$ modulo any power of 5 or 7 . In Hardy's extraction [83], he considerably amplified Ramanujan's arguments. Similarly, Rushforth [92] provided many details omitted by Ramanujan. In his paper providing proofs of the general congruences modulo $5^{n}$ and $7^{[n / 2]+1}$, Watson [108] had to supply most of the details omitted by Ramanujan. We have followed their leads and have supplied more details for some of Ramanujan's arguments. However, for those parts of the manuscript 
examined by Hardy, Rushforth, and Watson, we have not added details here, as readers can find these in the aforementioned papers. So that readers remain clear about what was written by Ramanujan, we have placed our additions in square brackets.

5) We have taken the liberty of making minor editorial changes without comments. Such alterations include correcting misprints, adding punctuation, and introducing notation. In particular, Ramanujan generally wrote infinite series in expanded form without resorting to summation signs, which we have supplied.

Many unproved claims can be found in the manuscript. Since Ramanujan's death, some have been proved by others, often without realizing that Ramanujan had originally found them. Some claims are false, and others had not been proved. Because of the desire to make minimal additions within Ramanujan's manuscript, we have deferred discussions of most of Ramanujan's unproved claims to the end of this paper, where many references to the literature are cited.

\section{PROPERTIES OF $p(n)$ AND $\tau(n)$ DEFINED BY THE FUNCTIONS$$
\begin{aligned}
& \sum_{n=0}^{\infty} p(n) q^{n}=(q ; q)_{\infty}^{-1}, \\
& \sum_{n=1}^{\infty} \tau(n) q^{n}=q(q ; q)_{\infty}^{24}
\end{aligned}
$$

\section{S. RAMANUJAN}

\section{I}

0. I have shown elsewhere by very simple arguments that

$$
\begin{aligned}
& p(5 n-1) \equiv 0(\bmod 5), \\
& p(7 n-2) \equiv 0(\bmod 7) .
\end{aligned}
$$

In the case of $\tau(n)$ such simple arguments give the following results.

\section{Modulus 2}

It is easy to see that the coefficients of $q^{n}$ in the expansion of

$$
q(q ; q)_{\infty}^{24} \quad \text { and } \quad q\left(q^{8} ; q^{8}\right)_{\infty}^{3}
$$

are both odd or both even, [where here and in the sequel

$$
(a ; q)_{\infty}=\prod_{n=0}^{\infty}\left(1-a q^{n}\right),
$$

where $|q|<1$.] But [by Jacobi's identity [51, p. 285, Thm. 357], [21, p. 39, Entry $24($ ii) $]$,

$$
q\left(q^{8} ; q^{8}\right)_{\infty}^{3}=\sum_{n=0}^{\infty}(-1)^{n}(2 n+1) q^{(2 n+1)^{2}} .
$$


It follows that $\tau(n)$ is odd or even according as $n$ is an odd square or not. Thus we see that the number of values of $n$ not exceeding $n$ for which $\tau(n)$ is odd is only

$$
\left[\frac{1+\sqrt{n}}{2}\right]
$$

\section{Modulus 5}

Further let $J$ be any function of $q$ with integral coefficients but not the same function throughout. It is easy to see that

$$
q(q ; q)_{\infty}^{24}=q(q ; q)_{\infty}^{4}\left(q^{5} ; q^{5}\right)_{\infty}^{4}+5 J .
$$

But the coefficient of $q^{5 n}$ in

$$
q(q ; q)_{\infty}^{4}
$$

is a multiple of $5 .^{1}$ It follows that

$$
\tau(5 n) \equiv 0 \quad(\bmod 5)
$$

\section{Modulus 7}

This is the simplest of all cases. Here we have

$$
q(q ; q)_{\infty}^{24}=q(q ; q)_{\infty}^{3}\left(q^{7} ; q^{7}\right)_{\infty}^{3}+7 J .
$$

But since

$$
q(q ; q)_{\infty}^{3}=q \sum_{n=0}^{\infty}(-1)^{n}(2 n+1) q^{n(n+1) / 2},
$$

it is easy to see that the coefficients of $q^{7 n}, q^{7 n-1}, q^{7 n-2}$ and $q^{7 n-4}$ are all multiples of 7 . It follows that

$$
\tau(7 n), \tau(7 n-1), \tau(7 n-2), \tau(7 n-4) \equiv 0 \quad(\bmod 7) .
$$

\section{Modulus 23}

We have

$$
q(q ; q)_{\infty}^{24}=q(q ; q)_{\infty}\left(q^{23} ; q^{23}\right)_{\infty}+23 J
$$

But [by Euler's pentagonal number theorem [51, p. 284, Thm. 353], [21, Entry 22(iii)]],

$$
q(q ; q)_{\infty}=\sum(-1)^{\nu} q^{1+\frac{1}{2} \nu(3 \nu+1)}
$$

where the summation extends over all values of $\nu$ from $-\infty$ to $\infty$. Now

$$
1+\frac{1}{2} \nu(3 \nu+1)=(6 \nu+1)^{2}-\frac{23 \nu(3 \nu+1)}{2} .
$$

\footnotetext{
${ }^{1}$ Recall that $p(5 n+4) \equiv 0(\bmod 5)$.
} 
The residues of a square number for modulus 23 cannot be

$$
5,7,10,11,14,15,17,19,20,21,22 .
$$

It follows from this that

$$
\left\{\begin{array}{l}
\tau(23 n-1), \tau(23 n-2), \tau(23 n-3), \tau(23 n-4), \\
\tau(23 n+5), \tau(23 n-6), \tau(23 n+7), \tau(23 n-8), \quad \equiv 0(\bmod 23), \\
\tau(23 n-9), \tau(23 n+10), \tau(23 n+11)
\end{array}\right.
$$

\section{Modulus 5}

1. Let

$$
\begin{aligned}
& P:=1-24 \sum_{n=1}^{\infty} \frac{n q^{n}}{1-q^{n}} \\
& Q:=1+240 \sum_{n=1}^{\infty} \frac{n^{3} q^{n}}{1-q^{n}}
\end{aligned}
$$

and

$$
R:=1-504 \sum_{n=1}^{\infty} \frac{n^{5} q^{n}}{1-q^{n}},
$$

so that ${ }^{2}$

$$
Q^{3}-R^{2}=1728 q(q ; q)_{\infty}^{24}
$$

Let $\sigma_{s}(n)$ denote the [sum of the] $s^{\text {th }}$ powers of the divisors of $n$. Then it is easy to see that

$$
Q=1+5 J ; \quad R=P+5 J
$$

Hence,

$$
Q^{3}-R^{2}=Q-P^{2}+5 J
$$

But $^{3}$

$$
Q-P^{2}=288 \sum_{n=1}^{\infty} n \sigma_{1}(n) q^{n}
$$

and it is obvious that

$$
(q ; q)_{\infty}^{24}=\frac{\left(q^{25} ; q^{25}\right)_{\infty}}{(q ; q)_{\infty}}+5 J
$$

\footnotetext{
${ }^{2}$ For an elementary proof, see [80, eq. (44)].

${ }^{3}$ See [80, eq. (36)].
} 
It follows from (1.1) and (1.3)-(1.5), that

$$
q \frac{\left(q^{25} ; q^{25}\right)_{\infty}}{(q ; q)_{\infty}}=\sum_{n=1}^{\infty} n \sigma_{1}(n) q^{n}+5 J .
$$

In other words

$$
\left(q^{25} ; q^{25}\right)_{\infty} \sum_{n=0}^{\infty} p(n) q^{n+1}=\sum_{n=1}^{\infty} n \sigma_{1}(n) q^{n}+5 J .
$$

But the coefficient of $q^{5 n}$ in the right hand side is a multiple of 5 . It follows that

$$
p(5 n-1) \equiv 0 \quad(\bmod 5) .
$$

It also follows from (1.7) that

$$
\begin{aligned}
& p(n-1)-p(n-26)-p(n-51)+p(n-126) \\
+ & p(n-176)-p(n-301)-\cdots-n \sigma_{1}(n) \equiv 0(\bmod 5),
\end{aligned}
$$

where $1,26,51,126, \ldots$ are numbers of the form $\frac{1}{2}(5 \nu+1)(15 \nu+2)$ and $\frac{1}{2}(5 \nu-1)(15 \nu-2)$. The number of values of $n$ not exceeding 200 for which $p(n) \equiv$ $0,1,2,3,4(\bmod 5)$ is $69,33,34,34,30$, respectively; and the least value of $n$ for which $p(n) \equiv 4(\bmod 5)$ is 30 . These being so it appears that $p(n) \equiv 0(\bmod 5)$ for about $\frac{1}{3}$ of the values of $n$ while $p(n) \equiv 1,2,3$ or $4(\bmod 5)$ for about $\frac{1}{6}$ of the values of $n$ each. It seems extremely difficult to prove any result in this direction concerning $p(n)$, but the problem is much easier concerning $\tau(n)$.

2. It follows from (1.5) and (1.6) that

$$
\left\{\begin{array}{l}
\tau(n)-n \sigma_{1}(n) \equiv 0(\bmod 5) \\
\lambda(n)-n \sigma_{1}(n) \equiv 0(\bmod 5)
\end{array}\right.
$$

where

$$
\sum_{n=1}^{\infty} \lambda(n) q^{n}=q \frac{\left(q^{25} ; q^{25}\right)_{\infty}}{(q ; q)_{\infty}}
$$

so that $\lambda(n+1)$ is the number of partitions of $n$ as the sum of integers which are not multiples of 25 . But if $n$ be written in the form

$$
2^{a_{2}} \cdot 3^{a_{3}} \cdot 5^{a_{5}} \cdot 7^{a_{7}} \cdots
$$

where the $a$ 's are zeroes or positive integers, then

$$
n \sigma_{1}(n)=\prod_{p} \frac{p^{a_{p}}\left(p^{1+a_{p}}-1\right)}{p-1}, \quad p=2,3,5, \ldots
$$

But

$$
\frac{p^{a_{p}}\left(p^{1+a_{p}}-1\right)}{p-1} \equiv 0 \quad(\bmod 5)
$$


if

$$
a_{p} \geq 1, \quad p=5
$$

or

$$
a_{p} \equiv 1(\bmod 2), \quad p \equiv 4(\bmod 5),
$$

or

$$
a_{p} \equiv 3(\bmod 4), \quad p \equiv 2 \text { or } 3(\bmod 5),
$$

or

$$
a_{p} \equiv 4(\bmod 5), \quad p \equiv 1(\bmod 5),
$$

and for no other values. Suppose now that

$$
\begin{cases}t_{n}=0, & \tau(n) \equiv 0(\bmod 5), \\ t_{n}=1, & \tau(n) \not \equiv 0(\bmod 5) .\end{cases}
$$

Then it follows from (2.3) that

$$
\sum_{n=1}^{\infty} \frac{t_{n}}{n^{s}}=\prod_{1} \prod_{2} \prod_{3}
$$

where

$$
\prod_{1}=\prod_{p} \frac{1}{1-p^{-2 s}}
$$

$p$ being a prime of the form $5 k-1$ and

$$
\prod_{2}=\prod_{p} \frac{1-p^{-3 s}}{\left(1-p^{-s}\right)\left(1-p^{-4 s}\right)},
$$

$p$ being a prime of the form $5 k \pm 2$ and

$$
\prod_{3}=\prod_{p} \frac{1-p^{-4 s}}{\left(1-p^{-s}\right)\left(1-p^{-5 s}\right)},
$$

$p$ being a prime of the form $5 k+1$.

It is easy to prove from (2.5) that

$$
\sum_{k=1}^{n} t_{k}=o(n)
$$

It can be shown by transcendental methods that

$$
\sum_{k=1}^{n} t_{k} \sim \frac{C n}{(\log n)^{1 / 4}}
$$


and

$$
\sum_{k=1}^{n} t_{k}=C \int_{1}^{n} \frac{d x}{(\log x)^{1 / 4}}+O\left(\frac{n}{(\log n)^{r}}\right)
$$

where $C$ is a constant and $r$ is any positive number.

The proof of (2.6) is quite elementary and very similar to that for showing that $\pi(x)=o(x),{ }^{4} \pi(x)$ being the number of primes not exceeding $x$. The result $(2.6)$ can be stated roughly in other words that $\tau(n)$ and $\lambda(n)$ are divisible by 5 for almost all values of $n$, while (2.7) and (2.8) give a lot more information.

\section{Modulus 25}

3. It is easily seen from (1.2) that

$$
\begin{aligned}
Q^{3}-R^{2} & =2\left(Q^{2}-P R\right)-\left(Q-P^{2}\right)+Q(Q-1)^{2}-(R-P)^{2} \\
& =2\left(Q^{2}-P R\right)-\left(Q-P^{2}\right)+25 J
\end{aligned}
$$

$\mathrm{But}^{5}$

$$
Q^{2}-P R=1008 \sum_{n=1}^{\infty} n \sigma_{5}(n) q^{n}
$$

and it is obvious that

$$
(q ; q)_{\infty}^{24}=\frac{\left(q^{5} ; q^{5}\right)_{\infty}^{5}}{(q ; q)_{\infty}}+25 J
$$

Now remembering that

$$
\sigma_{5}(n)-\sigma_{1}(n) \equiv 0 \quad(\bmod 5),
$$

it follows from (1.4) and (3.1)-(3.3) that

$$
6 q \frac{\left(q^{5} ; q^{5}\right)_{\infty}^{5}}{(q ; q)_{\infty}}=\sum_{n=1}^{\infty}\left\{2 n \sigma_{5}(n)-n \sigma_{1}(n)\right\} q^{n}+25 J
$$

[By extracting those terms with exponents that are multiples of 5 and by employing the congruence $p(5 n-1) \equiv 0(\bmod 5)$,] we easily deduce that

$$
(q ; q)_{\infty}^{5} \sum_{n=1}^{\infty} p(5 n-1) q^{n}=\sum_{n=1}^{\infty}\left\{10 n \sigma_{5}(n)-5 n \sigma_{1}(n)\right\} q^{n}+25 J
$$

and hence [by (3.4)] that

$$
\left(q^{5} ; q^{5}\right)_{\infty} \sum_{n=1}^{\infty} p(5 n-1) q^{n}=5 \sum_{n=1}^{\infty} n \sigma_{1}(n) q^{n}+25 J .
$$

\footnotetext{
${ }^{4}$ See Landau's Primzahlen [63, pp. 641-669].

${ }^{5}$ See [80, Table II].
} 
Since the coefficient of $q^{5 n}$ is a multiple of 25 it follows that

$$
p(25 n-1) \equiv 0 \quad(\bmod 25) .
$$

It also follows from (3.6) that

$$
\begin{aligned}
& p(5 n-1)-p(5 n-26)-p(5 n-51)+p(5 n-126) \\
+ & p(5 n-176)-\cdots-5 n \sigma_{1}(n) \equiv 0(\bmod 25),
\end{aligned}
$$

where $1,26,51,126, \ldots$ are the same as in (1.9).

4. It is easy to see [by Fermat's little theorem] that

$$
n \sigma_{9}(n)-2 n \sigma_{5}(n)+n \sigma_{1}(n) \equiv 0 \quad(\bmod 25) .
$$

It follows from this and (3.3) and (3.5) that

$$
\tau(n)-n \sigma_{9}(n) \equiv 0 \quad(\bmod 25) .
$$

It appears that, if $k$ be any positive integer, it is possible to find two integers $a$ and $b$ such that

$$
\tau(n)-n^{a} \sigma_{b}(n) \equiv 0 \quad\left(\bmod 5^{k}\right),
$$

if $n$ is not a multiple of 5 . Thus for instance

$$
\tau(n)-n^{41} \sigma_{29}(n) \equiv 0 \quad(\bmod 125),
$$

if $n$ is not a multiple of 5 . I have not yet proved these results. If $n$ is a multiple of 5 , then

$$
\tau(n)-4830 \tau\left(\frac{n}{5}\right)+5^{11} \tau\left(\frac{n}{25}\right)=0
$$

in virtue of (7.6), $\tau(x)$ being considered as 0 if $x$ is not an integer.

It also appears that the coefficient of $q^{n}$ in the left hand side of (3.5) can be exactly determined in terms of the real divisors of $n$. Thus

$$
q \frac{\left(q^{5} ; q^{5}\right)_{\infty}^{5}}{(q ; q)_{\infty}}=\sum_{n=1}^{\infty}\left(\frac{n}{5}\right) \frac{q^{n}}{\left(1-q^{n}\right)^{2}}
$$

[where $\left(\frac{n}{p}\right)$ denotes the Legendre symbol]. The allied function

$$
\frac{(q ; q)_{\infty}^{5}}{\left(q^{5} ; q^{5}\right)_{\infty}}=1-5 \sum_{n=1}^{\infty}\left(\frac{n}{5}\right) \frac{n q^{n}}{1-q^{n}}
$$

It follows from (4.5) that

$$
(q ; q)_{\infty}^{5} \sum_{n=1}^{\infty} p(5 n-1) q^{n}=5 \sum_{n=1}^{\infty}\left(\frac{n}{5}\right) \frac{q^{n}}{\left(1-q^{n}\right)^{2}}
$$


and hence that ${ }^{6}$

$$
\sum_{n=0}^{\infty} p(5 n+4) q^{n}=5 \frac{\left(q^{5} ; q^{5}\right)_{\infty}^{5}}{(q ; q)_{\infty}^{6}}
$$

\section{Modulus 7}

5. Since $^{7}$

$$
Q^{2}=1+480 \sum_{n=1}^{\infty} \frac{n^{7} q^{n}}{1-q^{n}}
$$

it is easy to see that

$$
Q^{2}=P+7 J ; \quad R=1+7 J
$$

and so

$$
\left(Q^{3}-R^{2}\right)^{2}=P^{3}-2 P Q+R+7 J .
$$

But $^{8}$

$$
\left\{\begin{array}{c}
P Q-R=720 \sum_{n=1}^{\infty} n \sigma_{3}(n) q^{n} \\
P^{3}-3 P Q+2 R=-1728 \sum_{n=1}^{\infty} n^{2} \sigma_{1}(n) q^{n}
\end{array}\right.
$$

and it is obvious that

$$
(q ; q)_{\infty}^{48}=\frac{\left(q^{49} ; q^{49}\right)_{\infty}}{(q ; q)_{\infty}}+7 J
$$

It follows from all these that

$$
q^{2} \frac{\left(q^{49} ; q^{49}\right)_{\infty}}{(q ; q)_{\infty}}=\sum_{n=1}^{\infty}\left\{n^{2} \sigma_{1}(n)-n \sigma_{3}(n)\right\} q^{n}+7 J
$$

In other words

$$
\left(q^{49} ; q^{49}\right)_{\infty} \sum_{n=0}^{\infty} p(n) q^{n+2}=\sum_{n=1}^{\infty}\left\{n^{2} \sigma_{1}(n)-n \sigma_{3}(n)\right\} q^{n}+7 J .
$$

It follows that

$$
p(7 n-2) \equiv 0 \quad(\bmod 7),
$$

${ }^{6}$ For a direct proof of this result see [81].

${ }^{7}$ See $[80$, Table I].

${ }^{8}$ See [80, Tables II and III, resp.]. 
and

$$
\begin{gathered}
p(n-2)-p(n-51)-p(n-100)+p(n-247) \\
+p(n-345)-\cdots+n \sigma_{3}(n)-n^{2} \sigma_{1}(n) \equiv 0(\bmod 7),
\end{gathered}
$$

where $2,51,100,247, \ldots$ are the numbers of the form $\frac{1}{2}(7 \nu+1)(21 \nu+4)$ and $\frac{1}{2}(7 \nu-1)(21 \nu-4)$.

The number of values of $n$ not exceeding 200 for which $p(n) \equiv 0,1,2,3,4,5,6$ $(\bmod 7)$ is $50,33,22,28,23,23,21$, respectively, and the least value of $n$ for which $p(n) \equiv 6(\bmod 7)$ is 73 . It appears that $p(n) \equiv 0(\bmod 7)$ for about $\frac{1}{4}$ of the values of $n$ while $p(n) \equiv 1,2,3,4,5,6(\bmod 7)$ for about $\frac{1}{8}$ of the values of $n$ each.

6. It follows from (5.2) that

$$
Q^{3}-R^{2}=P Q-R+7 J
$$

It is easy to see from this and (5.4) that

$$
\tau(n)-n \sigma_{3}(n) \equiv 0 \quad(\bmod 7) .
$$

Now if $n=2^{a_{2}} \cdot 3^{a_{3}} \cdot 5^{a_{5}} \cdot 7^{a_{7}} \cdots$, then

$$
n \sigma_{3}(n)=\prod_{p} p^{a_{p}} \frac{p^{3\left(1+a_{p}\right)}-1}{p^{3}-1}, \quad p=2,3,5,7, \ldots
$$

But

$$
p^{a_{p}} \frac{p^{3\left(1+a_{p}\right)}-1}{p^{3}-1} \equiv 0 \quad(\bmod 7)
$$

if

$$
a_{p} \equiv 6 \quad(\bmod 7), \quad p \equiv 1,2, \text { or } 4 \quad(\bmod 7)
$$

or

$$
a_{p} \equiv 1 \quad(\bmod 2), \quad p \equiv 3,5, \text { or } 6 \quad(\bmod 7)
$$

or

$$
a_{p} \geq 1, \quad p=7 \text {. }
$$

Suppose now that

$$
\begin{aligned}
& t_{n}=1, \quad \tau(n) \not \equiv 0(\bmod 7), \\
& t_{n}=0, \quad \tau(n) \equiv 0(\bmod 7) \text {. }
\end{aligned}
$$

Then it follows from (6.4) that

$$
\sum_{n=1}^{\infty} \frac{t_{n}}{n^{s}}=\prod_{1} \prod_{2}
$$

where

$$
\prod_{1}=\prod_{p} \frac{1-p^{-6 s}}{\left(1-p^{-s}\right)\left(1-p^{-7 s}\right)}
$$


$p$ being a prime of the form $7 k+1,7 k+2,7 k+4$, and

$$
\prod_{2}=\prod_{p} \frac{1}{1-p^{-2 s}}
$$

$p$ being a prime of the form $7 k+3,7 k+5,7 k+6$. It is easy to prove from (6.5) by quite elementary methods that

$$
\sum_{k=1}^{n} t_{k}=o(n)
$$

It can be shown by transcendental methods that

$$
\sum_{k=1}^{n} t_{k} \sim \frac{C n}{(\log n)^{1 / 2}}
$$

and

$$
\sum_{k=1}^{n} t_{k}=C \int_{1}^{n} \frac{d x}{(\log x)^{1 / 2}}+O\left(\frac{n}{(\log n)^{r}}\right)
$$

where $r$ is any positive number and

$$
\begin{aligned}
C= & \frac{6^{1 / 2}}{7^{3 / 4}} \frac{1-2^{-6}}{1-2^{-7}} \frac{1-11^{-6}}{1-11^{-7}} \frac{1-23^{-6}}{1-23^{-7}} \frac{1-29^{-6}}{1-29^{-7}} \cdots \\
& \times \frac{1}{\left\{\left(1-3^{-2}\right)\left(1-5^{-2}\right)\left(1-13^{-2}\right)\left(1-17^{-2}\right)\left(1-19^{-2}\right) \cdots\right\}^{1 / 2}},
\end{aligned}
$$

$2,11,23, \ldots$ being primes of the form $7 k+1,7 k+2$, and $7 k+4$ while $3,5,13$, $\ldots$ being primes of the form $7 k+3,7 k+5$ and $7 k+6$. Thus we see that $\tau(n)$ is divisible by 7 for almost all values of $n$; and at the same time the number of values of $n$ for which $\tau(n)$ is divisible by 7 is far more numerous than that for which $\tau(n)$ is divisible by 5 .

Now if

$$
\sum_{n=1}^{\infty} \lambda(n) q^{n}=q^{2} \frac{\left(q^{49} ; q^{49}\right)_{\infty}}{(q ; q)_{\infty}}
$$

so that $\lambda(n+2)$ is the number of partitions of $n$ as the sum of integers which are not multiples of 49 , it is clear from (5.6) that

$$
\lambda(n)-n^{2} \sigma_{1}(n)+n \sigma_{3}(n) \equiv 0 \quad(\bmod 7) .
$$

But it is easy to show that $n^{2} \sigma_{1}(n)$ and $n \sigma_{3}(n)$ are divisible by 7 for almost all values of $n$. It follows that $\lambda(n)$ is divisible by 7 for almost all values of $n$. It can even be shown that the number of values of $j$ not exceeding $n$ for which $\lambda(j)$ is not divisible by $j$ is

$$
O\left(\frac{n}{(\log n)^{1 / 6}}\right)
$$


The index $\frac{1}{6}$ in (6.9) is easily obtained by considering $n^{2} \sigma_{1}(n)$ and $n \sigma_{3}(n)$ separately; but whether this is the right index or not can be known only by considering

$$
n^{2} \sigma_{1}(n)-n \sigma_{3}(n)
$$

taken together, which seems rather complicated to deal with.

\section{Modulus 49}

7. We have

$$
\begin{aligned}
\left(Q^{3}-R^{2}\right)^{2}= & \left(3 P^{2} Q^{2}-4 P Q R-2 Q^{3}+3 R^{2}\right) \\
& -2\left(P^{3}-2 P Q+R\right)+2 P\left(Q^{2}-P\right)^{2}-(1+2 P Q)(R-1)^{2} \\
& +\left\{Q\left(Q^{2}-P\right)-R^{2}+1\right\}^{2} \\
= & \left(3 P^{2} Q^{2}-4 P Q R-2 Q^{3}+3 R^{2}\right)-2\left(P^{3}-2 P Q+R\right)+49 J
\end{aligned}
$$

in virtue of (5.2). But $^{9}$

$$
\left\{\begin{array}{l}
Q^{3}-R^{2}=1728 \sum_{n=1}^{\infty} \tau(n) q^{n} \\
3 Q^{3}+2 R^{2}-5 P Q R=1584 \sum_{n=1}^{\infty} n \sigma_{9}(n) q^{n} \\
5 Q^{3}+4 R^{2}-18 P Q R+9 P^{2} Q^{2}=8640 \sum_{n=1}^{\infty} n^{2} \sigma_{7}(n) q^{n}
\end{array}\right.
$$

and it is obvious that

$$
(q ; q)_{\infty}^{48}=\frac{\left(q^{7} ; q^{7}\right)_{\infty}^{7}}{(q ; q)_{\infty}}+49 J
$$

Now remembering that

$$
\left\{\begin{array}{l}
\sigma_{7}(n)-\sigma_{1}(n) \equiv 0(\bmod 7) \\
\sigma_{9}(n)-\sigma_{3}(n) \equiv 0(\bmod 7)
\end{array}\right.
$$

it follows from the above equations and (5.4) that

$$
\begin{aligned}
& q^{2} \frac{\left(q^{7} ; q^{7}\right)_{\infty}^{7}}{(q ; q)_{\infty}}=\sum_{n=1}^{\infty}\left\{2 n \sigma_{9}(n)-4 n^{2} \sigma_{7}(n)\right. \\
+ & \left.2 n \sigma_{3}(n)-2 n^{2} \sigma_{1}(n)+2 \tau(n)\right\} q^{n}+49 J .
\end{aligned}
$$

From this [and (6.2)] we deduce that

$$
(q ; q)_{\infty}^{7} \sum_{n=0}^{\infty} p(7 n+5) q^{n+1}=\sum_{n=1}^{\infty}\left\{28 n \sigma_{3}(n)+2 \tau(7 n)\right\} q^{n}+49 J
$$

${ }^{9}$ See [80, eq. (44), Table II, Table III, resp.]. 
I have stated in my previous paper that ${ }^{10}$

$$
\sum_{n=1}^{\infty} \frac{\tau(n)}{n^{s}}=\prod_{p} \frac{1}{1-\tau(p) p^{-s}+p^{11-2 s}}
$$

where $p$ assumes all prime values. This has since been proved by Mr Mordell. ${ }^{11}$ Now by actual calculation we find that

$$
\tau(7) \equiv 14 \quad(\bmod 49) .
$$

It follows from this and (7.6) that

$$
\tau(7 n)-14 \tau(n) \equiv 0 \quad(\bmod 49) .
$$

It is easy to see from this and (7.5) that

$$
\left(q^{7} ; q^{7}\right)_{\infty}^{7} \sum_{n=0}^{\infty} p(7 n+5) q^{n+1}=7 \sum_{n=1}^{\infty} n \sigma_{3}(n) q^{n}+49 J .
$$

Now if

$$
n \equiv 3,5,6 \quad(\bmod 7),
$$

then $n$ must contain an odd power of a prime $p$ of the form $7 k+3,7 k+5$ or $7 k+6$ as a divisor since all perfect squares are of the form $7 k, 7 k+1,7 k+2$ or $7 k+4$; and so $\sigma_{3}(n)$ is divisible by $p^{3}+1$ which is divisible by 7 . Also it is obvious that if $n$ is a multiple of 7 then $n \sigma_{3}(n)$ is also divisible by 7 . It follows that if

$$
n \equiv 0,3,5,6 \quad(\bmod 7)
$$

then

$$
n \sigma_{3}(n) \equiv 0 \quad(\bmod 7)
$$

It is easy to see from this and (7.7) that

$$
p(49 n-2), p(49 n-9), p(49 n-16), p(49 n-30) \equiv 0 \quad(\bmod 49) .
$$

It also follows from (7.7) that

$$
\begin{aligned}
& p(7 n-2)-p(7 n-51)-p(7 n-100)+p(7 n-247) \\
+ & p(7 n-345)-\cdots-7 n \sigma_{3}(n) \equiv 0(\bmod 49)
\end{aligned}
$$

where $2,51,100,247, \ldots$ are the same as in $(5.9)$.

8. It appears that

$$
q(q ; q)_{\infty}^{3}\left(q^{7} ; q^{7}\right)_{\infty}^{3}+8 q^{2} \frac{\left(q^{7} ; q^{7}\right)_{\infty}^{7}}{(q ; q)_{\infty}}=\sum_{n=1}^{\infty}\left(\frac{n}{7}\right) q^{n} \frac{1+q^{n}}{\left(1-q^{n}\right)^{3}}
$$

\footnotetext{
$10[80$, eq. (101)].

${ }^{11}$ On Mr Ramanujan's empirical expansions of modular functions, Proc. Cambridge Philos. Soc. 19 (1919), 117-124. A simpler proof is given in Hardy's lectures [50].
} 
[where $\left(\frac{n}{7}\right)$ denotes the Legendre symbol], while the allied function

$$
49 q(q ; q)_{\infty}^{3}\left(q^{7} ; q^{7}\right)_{\infty}^{3}+8 \frac{(q ; q)_{\infty}^{7}}{\left(q^{7} ; q^{7}\right)_{\infty}}=8-7 \sum_{n=1}^{\infty}\left(\frac{n}{7}\right) \frac{n^{2} q^{n}}{1-q^{n}} .
$$

Now remembering that

$$
(q ; q)_{\infty}^{3}=\sum_{n=0}^{\infty}(-1)^{n}(2 n+1) q^{n(n+1) / 2}
$$

and picking out the terms $q^{7}, q^{14}, q^{21}, \ldots$ from both sides in (8.1) we obtain

$$
-7 q(q ; q)_{\infty}^{3}\left(q^{7} ; q^{7}\right)_{\infty}^{3}+8(q ; q)_{\infty}^{7} \sum_{n=1}^{\infty} p(7 n-2) q^{n}=49 \sum_{n=1}^{\infty}\left(\frac{n}{7}\right) q^{n} \frac{1+q^{n}}{\left(1-q^{n}\right)^{3}}
$$

the series in the right hand side being the same as that in (8.1). It follows from this and (8.1) that ${ }^{12}$

$$
\sum_{n=0}^{\infty} p(7 n+5) q^{n}=7 \frac{\left(q^{7} ; q^{7}\right)_{\infty}^{3}}{(q ; q)_{\infty}^{4}}+49 q \frac{\left(q^{7} ; q^{7}\right)_{\infty}^{7}}{(q ; q)_{\infty}^{8}}
$$

It also appears that if

$$
\sum_{n=1}^{\infty} \lambda(n) q^{n}=q(q ; q)_{\infty}^{3}\left(q^{7} ; q^{7}\right)_{\infty}^{3}
$$

then

$$
\sum_{n=1}^{\infty} \frac{\lambda(n)}{n^{s}}=\frac{1}{1+7^{1-s}} \prod_{1} \prod_{2}
$$

where

$$
\prod_{1}=\prod_{p} \frac{1}{1-p^{2-2 s}}
$$

$p$ being a prime of the form $7 k+3,7 k+5$, or $7 k+6$, and

$$
\prod_{2}=\prod_{p} \frac{1}{1+\left(2 p-a^{2}\right) p^{-s}+p^{2-2 s}}
$$

$p$ being a prime of the form $7 k+1,7 k+2$, or $7 k+4$ and $a$ and $b$ being integers such that $4 p=a^{2}+7 b^{2}$. Thus $\lambda(n)$ can be completely ascertained. It follows from this and (8.1) and (8.2) that the coefficients of $q^{n}$ in

$$
\frac{(q ; q)_{\infty}^{7}}{\left(q^{7} ; q^{7}\right)_{\infty}}, \quad q^{2} \frac{\left(q^{7} ; q^{7}\right)_{\infty}^{7}}{(q ; q)_{\infty}}
$$

\footnotetext{
${ }^{12}$ For a direct proof of this see $\S$. [Ramanujan evidently intended to give a proof of (8.3) elsewhere. In his paper [81], (8.3) is stated without proof. See the notes at the end of this paper for references to proofs of (8.3).]
} 
can be completely ascertained.

Now it is easy to see that

$$
3 n^{9}-2 n^{3} \equiv 0,1, \text { or }-1 \quad(\bmod 49),
$$

according as $n \equiv 0(\bmod 7), n \equiv 1,2,4(\bmod 7)$, or $n \equiv 3,5,6(\bmod 7)$. Also the coefficient of $q^{n}$ in $q(1+q) /(1-q)^{3}$ is $n^{2}$. Hence the right side in (8.1) can be written as

$$
\sum_{n=1}^{\infty}\left\{3 n^{2} \sigma_{7}(n)-2 n^{2} \sigma_{1}(n)\right\} q^{n}+49 J .
$$

It follows from this, (7.3), (7.4) and (8.1) that

$$
\tau(n)-3 \lambda(n)+n \sigma_{9}(n)+n \sigma_{3}(n) \equiv 0 \quad(\bmod 49),
$$

where $\lambda(n)$ is the same as in (8.4). From the formulae (8.4) and (8.6) all the residues of $\tau(n)$ for modulus 49 can be completely ascertained.

\section{Modulus 11}

9. In this case we start with the series ${ }^{13}$

$$
\left\{\begin{aligned}
1-264 \sum_{n=1}^{\infty} \frac{n^{9} q^{n}}{1-q^{n}} & =Q R, \\
691+65520 \sum_{n=1}^{\infty} \frac{n^{11} q^{n}}{1-q^{n}} & =441 Q^{3}+250 R^{2} .
\end{aligned}\right.
$$

It follows that

$$
Q R=1+11 J ; \quad Q^{3}-3 R^{2}=-2 P+11 J .
$$

It is easy to see from this that

$$
\begin{aligned}
\left(Q^{3}-R^{2}\right)^{5} & =\left(Q^{3}-3 R^{2}\right)^{5}-Q\left(Q^{3}-3 R^{2}\right)^{3}-R\left(Q^{3}-3 R^{2}\right)^{2}-5 Q R+11 J \\
& =P^{5}-3 P^{3} Q-4 P^{2} R-5 Q R+11 J .
\end{aligned}
$$

But $^{14}$

$$
\left\{\begin{array}{l}
P^{5}-10 P^{3} Q+20 P^{2} R-15 P Q^{2}+4 Q R=-20736 \sum_{n=1}^{\infty} n^{4} \sigma_{1}(n) q^{n} \\
P^{3} Q-3 P^{2} R+3 P Q^{2}-Q R=3456 \sum_{n=1}^{\infty} n^{3} \sigma_{3}(n) q^{n} \\
P^{2} R-2 P Q^{2}+Q R=-1728 \sum_{n=1}^{\infty} n^{2} \sigma_{5}(n) q^{n} \\
P Q^{2}-Q R=720 \sum_{n=1}^{\infty} n \sigma_{7}(n) q^{n}
\end{array}\right.
$$

\footnotetext{
${ }^{13}$ See [80, Table I].

${ }^{14}$ See [80, Table III, Table II].
} 
and it is obvious that

$$
(q ; q)_{\infty}^{120}=\frac{\left(q^{121} ; q^{121}\right)_{\infty}}{(q ; q)_{\infty}}+11 J
$$

It is easy to see from all these that

$$
q^{5} \frac{\left(q^{121} ; q^{121}\right)_{\infty}}{(q ; q)_{\infty}}=\sum_{n=1}^{\infty}\left\{-n^{4} \sigma_{1}(n)+3 n^{3} \sigma_{3}(n)+3 n^{2} \sigma_{5}(n)-5 n \sigma_{7}(n)\right\} q^{n}+11 J .
$$

It follows from this that

$$
p(11 n-5) \equiv 0 \quad(\bmod 11)
$$

and

$$
\begin{gathered}
p(n-5)-p(n-126)-p(n-247)+p(n-610)+p(n-852) \\
-\cdots+n^{4} \sigma_{1}(n)-3 n^{3} \sigma_{3}(n)-3 n^{2} \sigma_{5}(n)+5 n \sigma_{7}(n) \equiv 0(\bmod 11),
\end{gathered}
$$

where $5,126,247,610, \ldots$ are numbers of the form $\frac{1}{2}(11 \nu+2)(33 \nu+5)$ and $\frac{1}{2}(11 \nu-2)(33 \nu-5)$. It is only to prove the general result $(9.7)$ we require all the details in (9.3). But we don't require all these details in order to prove (9.6) and the proof can be very much simplified as follows: we have ${ }^{15}$

$$
q \frac{d P}{d q}=\frac{P^{2}-Q}{12}, \quad q \frac{d Q}{d q}=\frac{P Q-R}{3}, \quad q \frac{d R}{d q}=\frac{P R-Q^{2}}{2} .
$$

Now using (9.2) and (9.8) we can show that ${ }^{16}$

$$
\left(Q^{3}-R^{2}\right)^{5}=q \frac{d J}{d q}+11 J
$$

It follows from this and (9.4) that

$$
q^{5} \frac{\left(q^{121} ; q^{121}\right)_{\infty}}{(q ; q)_{\infty}}=q \frac{d J}{d q}+11 J
$$

Since the coefficient of $q^{11 n}$ in the right hand side is a multiple of 11 it follows that

$$
p(11 n-5) \equiv 0 \quad(\bmod 11)
$$

The number of values of $n$ not exceeding 200 for which $p(n) \equiv 0,1,2,3,4,5,6$, $7,8,9,10(\bmod 11)$ is $77,23,24,14,15,14,5,12,8,8,0$, respectively. Even though these values seem to be very irregular it appears from the residues of $p(n)$ for moduli 5 and 7 and also from the next section that $p(n) \equiv 0(\bmod 11)$ for about

\footnotetext{
${ }^{15}$ See [80, eq. (30)].

${ }^{16}$ As mentioned in the beginning, the $J$ 's are not the same functions.
} 
$\frac{1}{6}$ of the values of $n$ while $p(n) \equiv 1,2,3,4,5,6,7,8,9,10(\bmod 11)$ for about $\frac{1}{12}$ of the values of $n$ each.

10. Mr H. B. C. Darling observed the remarkable fact (before I began to write this paper) that $p(n)$ is divisible by 11 for 45 values of $n$ not exceeding 100 . This can be explained by the formula (9.7) and the congruency of

$$
n^{4} \sigma_{1}(n)-3 n^{3} \sigma_{3}(n)-3 n^{2} \sigma_{5}(n)+5 n \sigma_{7}(n)
$$

for modulus 11. It can be shown by quite elementary methods that (10.1) is divisible by 11 for almost all values of $n$. [A proof of this fact is sketched in Section 19.] It can even be shown that the number of values of $n$ not exceeding $n$ for which (10.1) is not divisible by 11 is

$$
O\left(\frac{n}{(\log n)^{1 / 10}}\right)
$$

by considering the divisibility of the four terms in (10.1) separately; but a better result can be found only by considering all the four terms in (10.1) taken together. The same remarks apply to the function $\lambda(n)$ defined by

$$
\sum_{n=1}^{\infty} \lambda(n) q^{n}=q^{5} \frac{\left(q^{121} ; q^{121}\right)_{\infty}}{(q ; q)_{\infty}}
$$

so that $\lambda(n+5)$ is the number of partitions of $n$ as the sum of integers which are not multiples of 121 ; that is to say $\lambda(n)$ is divisible by 11 for almost all values of $n$; and the number of values of $\lambda(n)$ not divisible by 11 is of the form (10.2). It appears from $(10.3)$ that the number of values of $n$ for which $p(n) \equiv 0(\bmod 11)$ cannot be so high as $45 \%$ if $n$ exceeds 120 . Thus the number of values of $p$ divisible by 11 is

$$
\begin{aligned}
45 \%, & 0<n \leq 40 \\
45 \%, & 40<n \leq 80 \\
45 \%, & 80<n \leq 120 \\
35 \%, & 120<n \leq 160 \\
22 \frac{1}{2} \%, & 160<n \leq 200 .
\end{aligned}
$$

It is also very remarkable that, in the table of the first 200 values of $p(n)$, there is not a single value of $p(n)$ of the form $11 k-1$. This is probably due to such a high percentage of the values of $p(n)$ divisible by 11 in the beginning.

I have not yet investigated completely the residues of $\tau(n)$ for modulus 11. But it appears that if

$$
\sum_{n=1}^{\infty} \lambda(n) q^{n}=q(q ; q)_{\infty}^{2}\left(q^{11} ; q^{11}\right)_{\infty}^{2}
$$

then

$$
\sum_{n=1}^{\infty} \frac{\lambda(n)}{n^{s}}=\frac{1}{1-11^{-s}} \prod_{p} \frac{1}{1-\lambda(p) p^{-s}+p^{1-2 s}}
$$


$p$ assuming all prime values except 11 , and that $\lambda(p)$ can be determined also. If that is so then the residues of $\tau(n)$ for modulus 11 can also be ascertained since it is easily seen that

$$
\tau(n)-\lambda(n) \equiv 0 \quad(\bmod 11) .
$$

Again it is easy to show by using (7.6) [and the values $\tau(2)=-24, \tau(3)=$ $252, \tau(5)=4830, \tau(7)=-16744$, and $\tau(11)=534612$, which can be found in a table in Ramanujan's paper [80], [85, p. 153]] that

$$
\begin{aligned}
\sum_{n=1}^{\infty} \frac{\tau(n)}{n^{s}}= & \frac{1}{1+2^{1-s}+2^{1-2 s}} \frac{1}{\left(1-3^{3-s}\right)^{2}} \frac{1}{\left(1-5^{2-s}\right)\left(1-5^{4-s}\right)} \\
& \times \frac{1}{\left(1+7^{2-s}\right)\left(1-7^{4-s}\right)} \frac{1}{1-11^{-s}} \cdots+11 j,
\end{aligned}
$$

where $j$ is a Dirichlet series of the form

$$
\sum \frac{a_{n}}{n^{s}}
$$

$a_{n}$ being an integer.

From this we can deduce a number of results such as

$$
\tau\left(2^{4 \lambda-1} n\right) \equiv 0 \quad(\bmod 11)
$$

if $n$ is an odd integer;

$$
\tau\left(3^{11 \lambda-1} n\right) \equiv 0 \quad(\bmod 11)
$$

if $n$ is not a multiple of 3 ;

$$
\tau\left(5^{5 \lambda-1} n\right) \equiv 0 \quad(\bmod 11)
$$

if $n$ is not a multiple of 5 ;

$$
\tau\left(7^{10 \lambda-1} n\right) \equiv 0 \quad(\bmod 11)
$$

if $n$ is not a multiple of 7 ;

$$
\tau\left(11^{\lambda} n\right)-\tau(n) \equiv 0 \quad(\bmod 11)
$$

and so on. [The five congruences above can be established by expanding the appropriate factors in (10.5a) in geometric series. For example, consider

$$
\begin{aligned}
\frac{1}{1+2^{1-s}+2^{1-2 s}} & =-\frac{i}{2^{1-s}+1-i}+\frac{i}{2^{1-s}+1+i} \\
& =-\frac{i}{1-i} \sum_{n=0}^{\infty}\left(\frac{2^{1-s}}{i-1}\right)^{n}+\frac{i}{1+i} \sum_{n=0}^{\infty}\left(\frac{2^{1-s}}{-i-1}\right)^{n} \\
& =i \sum_{n=0}^{\infty} 2^{n(1-s)} e^{-3 \pi i(n+1) / 4}-i \sum_{n=0}^{\infty} 2^{n(1-s)} e^{3 \pi i(n+1) / 4} .
\end{aligned}
$$


Since $\sin \{3 \pi(n+1) / 4\}=0$ if and only if $n \equiv-1(\bmod 4)$, the assertion $(10.61)$ follows from (10.5a).]

Even though (10.61)-(10.64) are very analogous to one another further equations are not necessarily quite similar to these; sometimes there are more than one equation and sometimes there are equations of the form

$$
\tau(19 n) \equiv 0 \quad(\bmod 11)
$$

if $n$ is not a multiple of 19 , and

$$
\tau(29 n) \equiv 0 \quad(\bmod 11)
$$

if $n$ is not a multiple of 29 .

It is very likely that the primes $19,29, \ldots$ occurring in equations like $(10.8)$ and (10.9) are such that the sum of their reciprocals is a divergent series. If this assertion is true then $\tau(n)$ is divisible by 11 for almost all values of $n$ which is easily seen from (10.2).

\section{Moduli 2 and 3}

11. [It will be convenient to introduce Ramanujan's theta-functions $\varphi(q)$ and $\psi(q)$, defined by

$$
\varphi(q):=\sum_{n=-\infty}^{\infty} q^{n^{2}}=\frac{(-q ;-q)_{\infty}}{(q ;-q)_{\infty}}
$$

and

$$
\psi(q):=\sum_{n=0}^{\infty} q^{n(n+1) / 2}=\frac{\left(q^{2} ; q^{2}\right)_{\infty}}{\left(q ; q^{2}\right)_{\infty}},
$$

where the product representations are easy consequences of Jacobi's triple product identity.]

Before we proceed to consider higher moduli we shall see what the analogous formulae are in the cases of moduli 2 and 3. It is easy to see that [by (11.1b)]

$$
\frac{\left(q^{4} ; q^{4}\right)_{\infty}}{(q ; q)_{\infty}}=\frac{\left(q^{2} ; q^{2}\right)_{\infty}}{\left(q ; q^{2}\right)_{\infty}}+2 J=\psi(q)+2 J
$$

It follows that

$$
p(n)-p(n-4)-p(n-8)+p(n-20)+p(n-28)-\cdots
$$

is odd or even according as $n$ is a triangular number or not, 4, 8, 20, . b being numbers of the form $2 \nu(3 \nu+1)$ and $2 \nu(3 \nu-1)$.

$p(n)$ is odd for 110 values of $n$ not exceeding 200 and even for 90 values of $n$ in the same range. Thus $p(n)$ seems to be odd for more values of $n$ than those for which $p(n)$ is even. 
If

$$
\sum_{n=0}^{\infty} \lambda(n) q^{n}=\frac{\left(q^{4} ; q^{4}\right)_{\infty}}{(q ; q)_{\infty}}
$$

so that $\lambda(n)$ is the number of partitions of $n$ as the sum of integers which are not multiples of 4 then [by (11.1) and (11.1b)] $\lambda(n)$ is odd or even according as $n$ is a triangular number or not.

Again we have

$$
\frac{\left(q^{9} ; q^{9}\right)_{\infty}}{(q ; q)_{\infty}}=\frac{\left(q^{3} ; q^{3}\right)_{\infty}^{3}}{(q ; q)_{\infty}}+3 J
$$

But it can be shown [23] that

$$
q \frac{\left(q^{9} ; q^{9}\right)_{\infty}^{3}}{\left(q^{3} ; q^{3}\right)_{\infty}}=\sum_{n=1}^{\infty} \chi_{0}(n) \frac{q^{n}}{1+q^{n}+q^{2 n}}
$$

[where $\chi_{0}(n)$ is the principal character modulo 3]. But the right hand side in (11.4) is of the form

$$
\sum_{n=1}^{\infty} \chi_{0}(n) \frac{q^{n}}{\left(1-q^{n}\right)^{2}}+3 J
$$

and the coefficient of $q^{3 n+1}$ in the above series is $\sigma_{1}(3 n+1)$. It follows from this and (11.3) and (11.4) that

$$
\frac{\left(q^{9} ; q^{9}\right)_{\infty}}{(q ; q)_{\infty}}=\sum_{n=0}^{\infty} \sigma_{1}(3 n+1) q^{n}+3 J .
$$

From this we easily deduce that

$$
\begin{aligned}
& p(n)-p(n-9)-p(n-18)+p(n-45)+p(n-63) \\
& -p(n-108)-\cdots-\sigma_{1}(3 n+1) \equiv 0(\bmod 3),
\end{aligned}
$$

where $9,18,45, \ldots$ are numbers of the form $\frac{9}{2} \nu(3 \nu+1)$ and $\frac{9}{2} \nu(3 \nu-1)$.

The number of values of $n$ not exceeding 200 for which $p(n) \equiv 0,1,2(\bmod 3)$ is $66,68,66$ respectively. Thus it appears that $p(n) \equiv 0,1,2(\bmod 3)$ for about $\frac{1}{3}$ of the number of values of $n$ each.

It follows from (11.5) that if

$$
\sum_{n=0}^{\infty} \lambda(n) q^{n}=\frac{\left(q^{9} ; q^{9}\right)_{\infty}}{(q ; q)_{\infty}}
$$

so that $\lambda(n)$ is the number of partitions of $n$ as the sum of integers which are not multiples of 9 , then

$$
\lambda(n)-\sigma_{1}(3 n+1) \equiv 0 \quad(\bmod 3) .
$$

Again the left hand side of (11.4) is of the form

$$
q(q ; q)_{\infty}^{24}+3 J
$$


while the right hand side of (11.4) is of the form

$$
\sum_{n=1}^{\infty} \frac{n^{2} q^{n}}{\left(1-q^{n}\right)^{2}}+3 J
$$

It follows that

$$
\tau(n)-n \sigma_{1}(n) \equiv 0 \quad(\bmod 3) .
$$

Suppose now that

$$
\begin{cases}t_{n}=0, & \lambda(n) \equiv 0(\bmod 3), \\ t_{n}=1, & \lambda(n) \not \equiv 0(\bmod 3),\end{cases}
$$

and that

$$
\begin{cases}T_{n}=0, & \tau(n) \equiv 0(\bmod 3), \\ T_{n}=1, & \tau(n) \neq \equiv 0(\bmod 3) .\end{cases}
$$

Then we can easily deduce from (11.7), (11.8), and (2.2) that

$$
\sum_{n=0}^{\infty} \frac{t_{n}}{(3 n+1)^{s}}=\sum_{n=0}^{\infty} \frac{T_{n}}{n^{s}}=\prod_{1} \prod_{2}
$$

where

$$
\prod_{1}=\prod_{p} \frac{1}{1-p^{-2 s}}
$$

$p$ assuming prime values of the form $3 k-1$ and

$$
\prod_{2}=\prod_{p} \frac{1+p^{-s}}{1-p^{-3 s}}
$$

$p$ assuming prime values of the form $3 k+1$. We easily deduce from this that

$$
\left\{\begin{array}{l}
\sum_{k=1}^{n} t_{k}=o(n), \\
\sum_{k=1}^{n} T_{k}=o(n) .
\end{array}\right.
$$

In other words $\lambda(n)$ and $\tau(n)$ are divisible by 3 for almost all values of $n$. We can show by transcendental methods that

$$
\left\{\begin{array}{l}
\sum_{k=1}^{n} t_{k}=C \int_{1}^{n} \frac{d x}{(\log x)^{1 / 2}}+O\left(\frac{n}{(\log n)^{r}}\right), \\
\sum_{k=1}^{n} T_{k}=\frac{C}{3} \int_{1}^{n} \frac{d x}{(\log x)^{1 / 2}}+O\left(\frac{n}{(\log n)^{r}}\right)
\end{array}\right.
$$


where $r$ is any positive number and

$$
C=\frac{2^{1 / 2}}{3^{1 / 4}} \frac{1-7^{-2}}{1-7^{-3}} \frac{1-13^{-2}}{1-13^{-3}} \frac{1-19^{-2}}{1-19^{-3}} \cdots \frac{1}{\left\{\left(1-2^{-2}\right)\left(1-5^{-2}\right)\left(1-11^{-2}\right) \cdots\right\}^{1 / 2}}
$$

in both cases, $2,5,11, \ldots$ being primes of the form $3 k-1$ and $7,13,19, \ldots$ being primes of the form $3 k+1$.

\section{Further properties of $\tau(n)$}

12. It is easy to see $[$ from $(11.1 b)]$ that

$$
(q ; q)_{\infty}^{24}=\frac{\left(q^{2} ; q^{2}\right)_{\infty}^{8}}{\left(q ; q^{2}\right)_{\infty}^{8}}+32 J=\psi^{8}(q)+32 J .
$$

But [21, p. 139, Ex. (ii)]

$$
q \psi^{8}(q)=\sum_{n=1}^{\infty} \frac{n^{3} q^{n}}{1-q^{2 n}}
$$

and

$$
\sum_{n=1}^{\infty} n^{4} q^{n}=\frac{q}{1-q^{2}}+16 J
$$

and

$$
\sum_{n=1}^{\infty} n^{8} q^{n}=\frac{q}{1-q^{2}}+32 J
$$

[since

$$
\sum_{n=1}^{\infty} n^{4} q^{n} \equiv 1 \cdot q+0 \cdot q^{2}+1 \cdot q^{3}+0 \cdot q^{4}+\cdots=\frac{q}{1-q^{2}} \quad(\bmod 16)
$$

as $n^{4} \equiv 0,1(\bmod 16)$, according as $n$ is even or odd, and

$$
\sum_{n=1}^{\infty} n^{8} q^{n} \equiv 1 \cdot q+0 \cdot q^{2}+1 \cdot q^{3}+0 \cdot q^{4}+\cdots=\frac{q}{1-q^{2}} \quad(\bmod 32)
$$

as $n^{8} \equiv 0,1(\bmod 32)$, according as $n$ is even or odd.] It is easy to see from all these that

$$
\left\{\begin{array}{l}
\tau(n)-n^{3} \sigma_{1}(n) \equiv 0(\bmod 16) \\
\tau(n)-n^{3} \sigma_{5}(n) \equiv 0(\bmod 32)
\end{array}\right.
$$

Again we have

$$
(q ; q)_{\infty}^{24}=\frac{\left(q^{3} ; q^{3}\right)_{\infty}^{9}}{(q ; q)_{\infty}^{3}}+27 J
$$

But it can be shown that [22, p. 143, Thm. 8.7]

$$
q \frac{\left(q^{3} ; q^{3}\right)_{\infty}^{9}}{(q ; q)_{\infty}^{3}}=\sum_{n=1}^{\infty} \frac{n^{2} q^{n}}{1+q^{n}+q^{2 n}}
$$


Now it is easy to see that

$$
\sum_{n=1}^{\infty} n^{3} q^{n}=\frac{q}{1+q+q^{2}}+9 J
$$

and

$$
\sum_{n=1}^{\infty} n^{9} q^{n}=\frac{q}{1+q+q^{2}}+27 J
$$

since

$\sum_{n=1}^{\infty} n^{3} q^{n} \equiv 1 \cdot q-1 \cdot q^{2}+0 \cdot q^{3}+1 \cdot q^{4}-1 \cdot q^{5}+0 \cdot q^{6}+\cdots=\frac{q-q^{2}}{1-q^{3}}=\frac{q}{1+q+q^{2}} \quad(\bmod 9)$,

as $n^{3} \equiv 0,1,-1(\bmod 9)$, according as $n \equiv 0,1,-1(\bmod 3)$, and

$\sum_{n=1}^{\infty} n^{9} q^{n} \equiv 1 \cdot q-1 \cdot q^{2}+0 \cdot q^{3}+1 \cdot q^{4}-1 \cdot q^{5}+0 \cdot q^{6}+\cdots=\frac{q}{1+q+q^{2}} \quad(\bmod 27)$

as $n^{9} \equiv 0,1,-1(\bmod 27)$, according as $\left.n \equiv 0,1,-1(\bmod 3).\right]$ It follows that

$$
\left\{\begin{array}{l}
\tau(n)-n^{2} \sigma_{1}(n) \equiv 0(\bmod 9) \\
\tau(n)-n^{2} \sigma_{7}(n) \equiv 0(\bmod 27)
\end{array}\right.
$$

It is easy to deduce from (2.1), (4.2), (12.1) and (12.3) that

$$
\left\{\begin{aligned}
\tau(n)-n \sigma_{1}(n) & \equiv 0(\bmod 30), \\
\tau(n)-n^{2} \sigma_{1}(n) & \equiv 0(\bmod 36), \\
\tau(n)-n^{3} \sigma_{1}(n) & \equiv 0(\bmod 48), \\
\tau(n)-n^{5} \sigma_{1}(n) & \equiv 0(\bmod 120),
\end{aligned}\right.
$$

$$
\left\{\begin{aligned}
\tau(n)-n \sigma_{3}(n) & \equiv 0(\bmod 42) \\
\tau(n)-n^{2} \sigma_{3}(n) & \equiv 0(\bmod 60), \\
\tau(n)-n^{4} \sigma_{3}(n) & \equiv 0(\bmod 168)
\end{aligned}\right.
$$

$$
\left\{\begin{aligned}
\tau(n)-n^{3} \sigma_{5}(n) & \equiv 0(\bmod 288), \\
\tau(n)-n^{2} \sigma_{7}(n) & \equiv 0(\bmod 540), \\
\tau(n)-n \sigma_{9}(n) & \equiv 0(\bmod 1050) .
\end{aligned}\right.
$$

Again it easily follows from the second equation in (9.1) that

$$
\tau(n)-\sigma_{11}(n) \equiv 0 \quad(\bmod 691) .
$$


It is easy to deduce from this that $\tau(n)$ is divisible by 691 for almost all values of $n$, and by transcendental methods that the number of values of $n$ not exceeding $n$ for which $\tau(n)$ is not divisible by 691 is of the form

$$
C \int_{1}^{n} \frac{d x}{(\log x)^{1 / 690}}+O\left(\frac{n}{(\log n)^{r}}\right)
$$

where $C$ is a constant and $r$ is any positive number.

It is easy to prove that

$$
q(-q ;-q)_{\infty}^{24}=q(q ; q)_{\infty}^{24}+48 q^{2}\left(q^{2} ; q^{2}\right)_{\infty}^{24}+2^{12} q^{4}\left(q^{4} ; q^{4}\right)_{\infty}^{24}
$$

[To prove (12.7b), set, after Ramanujan,

$$
f(-q):=(q ; q)_{\infty}
$$

Thus, (12.7b) can be written in the equivalent formulation

$$
q f^{24}(q)=q f^{24}(-q)+48 q^{2} f^{24}\left(-q^{2}\right)+2^{12} q^{4} f^{24}\left(-q^{4}\right) .
$$

To prove (12.7c), we use the catalogue of evaluations for $f$ found in Entry 12 of Chapter 17 in Ramanujan's second notebook [21, p. 124], in particular,

$$
\begin{aligned}
f(q) & =\sqrt{z} 2^{-1 / 6}\{x(1-x) / q\}^{1 / 24}, & & f(-q)=\sqrt{z} 2^{-1 / 6}(1-x)^{1 / 6}(x / q)^{1 / 24}, \\
f\left(-q^{2}\right) & =\sqrt{z} 2^{-1 / 3}\{x(1-x) / q\}^{1 / 12}, & & f\left(-q^{4}\right)=\sqrt{z} 2^{-2 / 3}(1-x)^{1 / 24}(x / q)^{1 / 6},
\end{aligned}
$$

where $x=k^{2}$, with $k$ being the modulus, and $z=(2 / \pi) K$, with $K$ being the complete elliptic integral of the first kind. Using these evaluations in (12.7c), we easily verify its truth.] From this it is easy to deduce that

$$
\tau(2 n)+24 \tau(n)+2^{11} \tau\left(\frac{1}{2} n\right)=0
$$

where $n$ is any integer and $\tau(x)=0$ if $x$ is not an integer.

[Recall that $\varphi$ and $\psi$ are defined in (11.1a) and (11.1b), respectively.] Again it is easy to prove that

$$
q \psi^{8}(q) \varphi^{16}(-q)=q f^{24}(-q) .
$$

[To prove this identity, use (12.7c) and the evaluations [21, Entry 11(i), p. 123; Entry 10(ii), p. 122]

$$
\left.\psi(q)=\sqrt{\frac{1}{2} z}(x / q)^{1 / 8} \quad \text { and } \quad \varphi(-q)=\sqrt{z}(1-x)^{1 / 4} .\right]
$$

But [by the binomial theorem],

$$
\varphi^{16}(-q)=-4 \varphi^{4}(-q)+16 \varphi^{2}(-q)-11+256 J .
$$

Hence

$$
\begin{aligned}
q f^{24}(-q) & =4\left\{1-\varphi^{4}(-q)\right\} q \psi^{8}(q)-16\left\{1-\varphi^{2}(-q)\right\} q \psi^{8}(q)+q \psi^{8}(q)+256 J \\
& =4\left\{1-\varphi^{4}(-q)\right\} q \psi^{4}\left(q^{2}\right)-16\left\{1-\varphi^{2}(-q)\right\} q \psi^{4}\left(q^{2}\right)+q \psi^{8}(q)+256 J
\end{aligned}
$$


But

$$
\begin{aligned}
q \psi^{8}(q) & =\sum_{n=0}^{\infty} \frac{n^{3} q^{n}}{1-q^{2 n}} \\
q \psi^{4}\left(q^{2}\right) & =\sum_{n=0}^{\infty} \frac{(2 n+1) q^{2 n+1}}{1-q^{4 n+2}}
\end{aligned}
$$

$$
\begin{aligned}
q \psi^{4}\left(q^{2}\right) \varphi^{4}(-q) & =\sum_{n=1}^{\infty}(-1)^{n-1} \frac{n^{3} q^{n}}{1-q^{2 n}} \\
q \psi^{4}\left(q^{2}\right) \varphi^{2}(-q) & =\sum_{n=1}^{\infty}(-1)^{n-1} \frac{n^{2} q^{n}}{1+q^{2 n}} \\
& =\sum_{n=0}^{\infty}(-1)^{n} \frac{(2 n+1)^{2} q^{2 n+1}}{1-q^{4 n+2}}-\sum_{n=1}^{\infty} \frac{(2 n)^{2} q^{2 n}}{1+q^{4 n}}+16 J
\end{aligned}
$$

[The identities (12.8b) and (12.8c) are, respectively, Examples (ii) and (iii) in Section 17 of Chapter 17 in Ramanujan's second notebook [21, p. 139].

By Entry 11(iii) in Chapter 17 of Ramanujan's second notebook [21, p. 123],

$$
\psi\left(q^{2}\right)=\frac{1}{2} \sqrt{z}(x / q)^{1 / 4} .
$$

It follows from (12.8a) and (12.8f) that

$$
q \psi\left(q^{2}\right) \varphi^{4}(-q)=\frac{1}{16} z^{4} x(1-x) .
$$

On the other hand by Entry 14(ii), (ix) in Chapter 17 of the second notebook [21, p. 130],

$$
\begin{aligned}
\sum_{n=1}^{\infty}(-1)^{n-1} \frac{n^{3} q^{n}}{1-q^{2 n}} & =\sum_{n=1}^{\infty}(-1)^{n-1} n^{3}\left(\frac{q^{n}}{1+q^{n}}+\frac{q^{2 n}}{1-q^{2 n}}\right) \\
& =\frac{1}{16}\left(1+\sum_{n=1}^{\infty}(-1)^{n-1} \frac{n^{3} q^{n}}{1+q^{n}}-1+16 \sum_{n=1}^{\infty}(-1)^{n-1} \frac{n^{3} q^{2 n}}{1-q^{2 n}}\right) \\
& =\frac{1}{16} z^{4} x(1-x) .
\end{aligned}
$$

The equality (12.8d) is now a trivial consequence of $(12.8 \mathrm{~g})$ and $(12.8 \mathrm{~h})$.

To prove (12.8e), first observe, by (12.8a) and (12.8f), that

$$
q \psi^{4}\left(q^{2}\right) \varphi^{2}(-q)=\frac{1}{16} z^{3} x \sqrt{1-x} .
$$

Next,

$$
\begin{aligned}
\sum_{n=1}^{\infty}(-1)^{n-1} \frac{n^{2} q^{n}}{1+q^{2 n}} & =-\sum_{n=1}^{\infty} \frac{4 n^{2} q^{2 n}}{1+q^{4 n}}+\sum_{n=1}^{\infty} \frac{(2 n+1)^{2} q^{2 n+1}}{1+q^{4 n+2}} \\
& =-8 \sum_{n=1}^{\infty} \frac{n^{2} q^{2 n}}{1+q^{4 n}}+\sum_{n=1}^{\infty} \frac{n^{2} q^{n}}{1+q^{2 n}} \\
& =-8 \sum_{n=1}^{\infty} \frac{n^{2} q^{2 n}}{1+q^{4 n}}+\frac{1}{16} z^{3} x
\end{aligned}
$$


by Entry 17(ii) in Chapter 17 of Ramanujan's second notebook [21, p. 138]. To evaluate the sum on the far right side of $(12.8 \mathrm{j})$, we apply the process of duplication [21, p. 125] to Entry 17(ii) cited above. Accordingly,

$$
\begin{aligned}
-8 \sum_{n=1}^{\infty} \frac{n^{2} q^{2 n}}{1+q^{4 n}} & =-\frac{1}{2}\left(\frac{1}{2} z(1+\sqrt{1-x})\right)^{3}\left(\frac{1-\sqrt{1-x}}{1+\sqrt{1-x}}\right)^{2} \\
& =-\frac{1}{16} z^{3} x(1-\sqrt{1-x}),
\end{aligned}
$$

after simplification. Putting $(12.8 \mathrm{k})$ into $(12.8 \mathrm{j})$, we readily find that

$$
\sum_{n=1}^{\infty}(-1)^{n-1} \frac{n^{2} q^{n}}{1+q^{2 n}}=\frac{1}{16} z^{3} x \sqrt{1-x} .
$$

Combining (12.8i) and (12.8k), we complete the proof of the first part of $(12.8 \mathrm{e})$.

To prove the second part of $(12.8 \mathrm{e})$, it clearly suffices to prove that

$$
S:=\sum_{n=0}^{\infty} \frac{(2 n+1)^{2} q^{2 n+1}}{1+q^{4 n+2}} \equiv \sum_{n=0}^{\infty}(-1)^{n} \frac{(2 n+1)^{2} q^{2 n+1}}{1-q^{4 n+2}}=: T \quad(\bmod 16)
$$

Now,

$$
\begin{aligned}
S & =\sum_{n=0}^{\infty} \frac{(2 n+1)^{2} q^{2 n+1}}{1-q^{4 n+2}}-2 \sum_{n=0}^{\infty} \frac{(2 n+1)^{2} q^{6 n+3}}{1-q^{8 n+4}} \\
& =T+2 \sum_{n=0}^{\infty} \frac{(4 n+3)^{2} q^{4 n+3}}{1-q^{8 n+6}}-2 \sum_{n=0}^{\infty} \frac{(2 n+1)^{2} q^{6 n+3}}{1-q^{8 n+4}} \\
& \equiv T+2 \sum_{n=0}^{\infty} \frac{q^{4 n+3}}{1-q^{8 n+6}}-2 \sum_{n=0}^{\infty} \frac{q^{6 n+3}}{1-q^{8 n+4}}(\bmod 16) \\
& =T+2 \sum_{n=0}^{\infty} \frac{q^{6 n+3}}{1-q^{8 n+4}}(\bmod 16)-2 \sum_{n=0}^{\infty} \frac{q^{6 n+3}}{1-q^{8 n+4}}(\bmod 16) \\
& =T(\bmod 16),
\end{aligned}
$$

where in the antipenultimate line above we expanded the summands of the first series in geometric series and then reversed the order of summation. This completes the proof of $(12.8 \mathrm{n})$, and hence the proof of the second equality of (12.8e).]

It follows from all these that

$$
\begin{aligned}
q(q ; q)_{\infty}^{24}= & -3 \sum_{n=0}^{\infty} \frac{(2 n+1)^{3} q^{2 n+1}}{1-q^{4 n+2}}+5 \sum_{n=1}^{\infty} \frac{(2 n)^{3} q^{2 n}}{1-q^{4 n}}-12 \sum_{n=0}^{\infty} \frac{(2 n+1) q^{2 n+1}}{1-q^{4 n+2}} \\
& +16 \sum_{n=0}^{\infty}(-1)^{n} \frac{(2 n+1)^{2} q^{2 n+1}}{1-q^{4 n+2}}-16 \sum_{n=1}^{\infty} \frac{(2 n)^{2} q^{2 n}}{1+q^{4 n}}+256 J .
\end{aligned}
$$

Now equating only the odd powers of $q$ we obtain

$$
\begin{aligned}
\sum_{n=0}^{\infty} \tau(2 n+1) q^{2 n+1}= & -3 \sum_{n=0}^{\infty} \frac{(2 n+1)^{3} q^{2 n+1}}{1-q^{4 n+2}}+16 \sum_{n=0}^{\infty}(-1)^{n} \frac{(2 n+1)^{2} q^{2 n+1}}{1-q^{4 n+2}} \\
& -12 \sum_{n=0}^{\infty} \frac{(2 n+1) q^{2 n+1}}{1-q^{4 n+2}}+256 J .
\end{aligned}
$$


But if $n$ be of the form $4 k+1$ then it is easy to see that

$$
n^{11}+3 n^{3}-16 n^{2}+12 n \equiv 0 \quad(\bmod 256) .
$$

Changing $n$ to $-n$ in this formula we see that if $n$ be of the form $4 k-1$ then

$$
n^{11}+3 n^{3}+16 n^{2}+12 n \equiv 0 \quad(\bmod 256) .
$$

It follows that

$$
\sum_{n=0}^{\infty} \tau(2 n+1) q^{2 n+1}=\sum_{n=0}^{\infty} \frac{(2 n+1)^{11} q^{2 n+1}}{1-q^{4 n+2}}+256 J
$$

In other words,

$$
\tau(n)-\sigma_{11}(n) \equiv 0 \quad(\bmod 256)
$$

for all odd values of $n$, while the formula (12.8) combined with this enable us to find the residues of $\tau(n)$ for modulus $2^{11}$ for even values of $n$. Thus

$$
\tau(n)+24 \sigma_{11}(n) \equiv 0 \quad(\bmod 2048)
$$

for all values of $n$.

It follows from (12.7) and (12.9) that

$$
\tau(n)-\sigma_{11}(n) \equiv 0 \quad(\bmod 176896)
$$

for all odd values of $n$.

\section{Modulus 13}

13. In this case we start with the second series in (9.1) and the series

$$
1-24 \sum_{n=1}^{\infty} \frac{n^{13} q^{n}}{1-q^{n}}=Q^{2} R
$$

It follows from these that

$$
Q^{3}-3 R^{2}=-2+13 J ; \quad Q^{2} R=P+13 J .
$$

Hence we have

$$
\begin{aligned}
\left(Q^{3}-R^{2}\right)^{7}= & -2\left(R^{2}-1\right)^{7}+13 J \\
= & -5 R^{6}\left(3 R^{2}-2\right)^{4}-2 R^{4}\left(3 R^{2}-2\right)^{3}+6 R^{4}\left(3 R^{2}-2\right)^{2} \\
& -6 R^{2}\left(3 R^{2}-2\right)^{2}-6 R^{2}\left(3 R^{2}-2\right)-2\left(R^{2}-1\right)+13 J \\
= & -5 P^{6}-2 P^{4} Q+6 P^{3} R-6 P^{2} Q^{2}-6 P Q R-\left(Q^{3}-R^{2}\right)+13 J .
\end{aligned}
$$


But $^{17}$

(13.3)

$$
\left\{\begin{array}{l}
5\left(P^{6}-15 P^{4} Q+40 P^{3} R-45 P^{2} Q^{2}+24 P Q R\right) \\
-\left(9 Q^{3}+16 R^{2}\right)=-248832 \sum_{n=1}^{\infty} n^{5} \sigma_{1}(n) q^{n} \\
7\left(P^{4} Q-4 P^{3} R+6 P^{2} Q^{2}-4 P Q R\right)+\left(3 Q^{3}+4 R^{2}\right)=41472 \sum_{n=1}^{\infty} n^{4} \sigma_{3}(n) q^{n} \\
2\left(P^{3} R-3 P^{2} Q^{2}+3 P Q R\right)-\left(Q^{3}+R^{2}\right)=-5184 \sum_{n=1}^{\infty} n^{3} \sigma_{5}(n) q^{n} \\
9(P Q-R)^{2}+5\left(Q^{3}-R^{2}\right)=8640 \sum_{n=1}^{\infty} n^{3} \sigma_{7}(n) q^{n} \\
5 P Q R-\left(3 Q^{3}+2 R^{2}\right)=-1584 \sum_{n=1}^{\infty} n \sigma_{9}(n) q^{n} \\
Q^{3}-R^{2}=1728 \sum_{n=1}^{\infty} \tau(n) q^{n} ;
\end{array}\right.
$$

and it is obvious that

$$
(q ; q)_{\infty}^{168}=\frac{\left(q^{169} ; q^{169}\right)_{\infty}}{(q ; q)_{\infty}}+13 J
$$

It is easy to see from all these that

$$
q^{7} \frac{\left(q^{169} ; q^{169}\right)_{\infty}}{(q ; q)_{\infty}}=\left(q^{169} ; q^{169}\right)_{\infty} \sum_{n=0}^{\infty} p(n) q^{n+7}
$$

$$
=\sum_{n=1}^{\infty}\left\{n^{5} \sigma_{1}(n)-4 n^{4} \sigma_{3}(n)-3 n^{3} \sigma_{5}(n)+6 n^{2} \sigma_{7}(n)-3 n \sigma_{9}(n)+3 \tau(n)\right\} q^{n}+13 J .
$$

It is easy to see by actual calculation that $\tau(13) \equiv 8(\bmod 13)$ in virtue of $(7.6)$ and hence $\tau(13 n)-8 \tau(n) \equiv 0(\bmod 13)$. It follows from this and $(13.5)$ that

$$
\sum_{n=1}^{\infty} p(13 n-7) q^{n}\left(q^{13} ; q^{13}\right)_{\infty}=11 \sum_{n=1}^{\infty} \tau(n) q^{n}+13 J
$$

It is not necessary to know all the details above in order to prove (13.6). The proof can be very much simplified as follows; using (9.8) and (13.2) we can show that

$$
\left(Q^{3}-R^{2}\right)^{7}=q \frac{d J}{d q}+3\left(Q^{3}-R^{2}\right)+13 J .
$$

\footnotetext{
${ }^{17}$ See [80], where not all these equalities are given, but where the same methods can be employed to provide proofs.
} 
It follows from this that

$$
q^{7} \frac{\left(q^{169} ; q^{169}\right)_{\infty}}{(q ; q)_{\infty}}=q \frac{d J}{d q}+3 \sum_{n=1}^{\infty} \tau(n) q^{n}+13 J .
$$

From this we easily deduce (13.6).

Again picking out the terms $q^{13}, q^{26}, q^{39}, \ldots$ in (13.6) we obtain [using the congruence $\tau(13 n) \equiv 8 \tau(n)(\bmod 13)]$

$$
\sum_{n=1}^{\infty} p\left(13^{2} n-7\right) q^{n}(q ; q)_{\infty}=10 \sum_{n=1}^{\infty} \tau(n) q^{n}+13 J .
$$

It follows from (13.5) that if

$$
\sum_{n=1}^{\infty} \lambda(n) q^{n}=q^{7} \frac{\left(q^{169} ; q^{169}\right)_{\infty}}{(q ; q)_{\infty}}
$$

so that $\lambda(n+7)$ is the number of partitions of $n$ as the sum of integers which are not multiples of 169 , then

$$
\begin{aligned}
& \lambda(n)-n^{5} \sigma_{1}(n)+4 n^{4} \sigma_{3}(n)+3 n^{3} \sigma_{5}(n) \\
& -6 n^{2} \sigma_{7}(n)+3 n \sigma_{9}(n)-3 \tau(n) \equiv 0(\bmod 13) .
\end{aligned}
$$

The results analogous to (10.61)-(10.9) in the case of modulus 13 are

$$
\tau\left(5^{12 \lambda-1} n\right) \equiv 0 \quad(\bmod 13)
$$

if $n$ is not a multiple of 5 ;

$$
\tau(7 n) \equiv 0 \quad(\bmod 13)
$$

if $n$ is not a multiple of 7 ;

$$
\tau(11 n) \equiv 0 \quad(\bmod 13)
$$

if $n$ is not a multiple of 11 ;

$$
\tau(13 n)-8 \tau(n) \equiv 0 \quad(\bmod 13)
$$

if $n$ is any integer;

$$
\tau\left(19^{4 \lambda-1} n\right) \equiv 0 \quad(\bmod 13)
$$

if $n$ is not a multiple of 19 ;

$$
\tau\left(23^{3 \lambda-1} n\right) \equiv 0 \quad(\bmod 13)
$$

if $n$ is not a multiple of 23 ;

$$
\tau\left(29^{6 \lambda-1} n\right) \equiv 0 \quad(\bmod 13)
$$

if $n$ is not a multiple of 29; and so on. 
14. The formulae (13.6) and (13.9) can be written as

$$
\sum_{n=0}^{\infty} p(13 n+6) q^{n}=11(q ; q)_{\infty}^{11}+13 J
$$

and

$$
\sum_{n=0}^{\infty} p\left(13^{2} n+162\right) q^{n}=23(q ; q)_{\infty}^{23}+13 J .
$$

Since I began to write this paper I have found by a different method that if $\lambda$ be any positive odd integer then

$$
\sum_{n=0}^{\infty} p\left(13^{\lambda} n+\frac{11 \cdot 13^{\lambda}+1}{24}\right) q^{n}=-2^{(5 \lambda-3) / 2}(q ; q)_{\infty}^{11}+13 J
$$

and if $\lambda$ be any positive even integer then

$$
\sum_{n=0}^{\infty} p\left(13^{\lambda} n+\frac{23 \cdot 13^{\lambda}+1}{24}\right) q^{n}=-2^{(5 \lambda-2) / 2}(q ; q)_{\infty}^{23}+13 J .
$$

I shall reserve the discussion of these results to another paper.

A number of results such as the following can be deduced from (14.3) and (14.4). [Note that

$$
(q ; q)_{\infty}^{11}=1-11 q+44 q^{2}-55 q^{3}-110 q^{4}+374 q^{5}-143 q^{6}+\cdots
$$

and

$$
\left.(q ; q)_{\infty}^{23}=1-23 q+230 q^{2}-1265 q^{3}+3795 q^{4}-3519 q^{5}-16445 q^{6}+\cdots .\right]
$$

If $\lambda$ be any positive odd integer then

$$
\begin{cases}p\left(\frac{11 \cdot 13^{\lambda}+1}{24}\right)+2^{(5 \lambda-3) / 2}, & p\left(\frac{35 \cdot 13^{\lambda}+1}{24}\right)+2^{(5 \lambda-1) / 2}, \\ p\left(\frac{59 \cdot 13^{\lambda}+1}{24}\right)-2^{(5 \lambda+3) / 2}, & p\left(\frac{83 \cdot 13^{\lambda}+1}{24}\right)-2^{5(\lambda+1) / 2}, \\ p\left(\frac{107 \cdot 13^{\lambda}+1}{24}\right)-2^{(5 \lambda+7) / 2}, & p\left(\frac{131 \cdot 13^{\lambda}+1}{24}\right)-2^{5(\lambda+1) / 2}, \\ p\left(\frac{155 \cdot 13^{\lambda}+1}{24}\right), & \end{cases}
$$

and so on are all divisible by 13 ; and if $\lambda$ be any positive even integer then

$$
\begin{cases}p\left(\frac{23 \cdot 13^{\lambda}+1}{24}\right)+2^{(5 \lambda-2) / 2}, & p\left(\frac{47 \cdot 13^{\lambda}+1}{24}\right)+2^{(5 \lambda+6) / 2}, \\ p\left(\frac{71 \cdot 13^{\lambda}+1}{24}\right)-2^{(5 \lambda+2) / 2}, & p\left(\frac{95 \cdot 13^{\lambda}+1}{24}\right)-2^{(5 \lambda+2) / 2}, \\ p\left(\frac{119 \cdot 13^{\lambda}+1}{24}\right)-2^{(5 \lambda-2) / 2}, & p\left(\frac{143 \cdot 13^{\lambda}+1}{24}\right)+2^{(5 \lambda+2) / 2}, \\ p\left(\frac{167 \cdot 13^{\lambda}+1}{24}\right), & \end{cases}
$$


and so on are all divisible by 13. In other words if $n$ is fixed and $\lambda+n$ is an even integer then the residue of

$$
p\left(\frac{13^{\lambda}(12 n-1)+1}{24}\right)
$$

for modulus 13 can be completely ascertained.

\section{General Theory}

\section{Modulus $\varpi$ where $\varpi$ is a prime greater than 3}

15. We start with the two series

$$
v_{\varpi-1}+(-1)^{(\varpi-1) / 2} 2(\varpi-1) \delta_{\varpi-1} \sum_{n=1}^{\infty} \frac{n^{\varpi-2} q^{n}}{1-q^{n}}=\sum K_{\ell, m}^{\prime} Q^{\ell} R^{m},
$$

where $K_{\ell, m}^{\prime}$ is a constant integer and the summation extends over all positive integral values of $\ell$ and $m$ (including zero) such that

$$
4 \ell+6 m=\varpi-1
$$

and

$$
v_{\varpi+1}+(-1)^{(\varpi+1) / 2} 2(\varpi+1) \delta_{\varpi+1} \sum_{n=1}^{\infty} \frac{n^{\varpi} q^{n}}{1-q^{n}}=\sum K_{\ell, m} Q^{\ell} R^{m},
$$

where $K_{\ell, m}$ is a constant integer and the summation extends over all positive integral values of $\ell$ and $m$ (including zero) such that

$$
4 \ell+6 m=\varpi+1 .
$$

In both the series $v_{s}$ and $\delta_{s}$ are the numerator and the denominator of $B_{s}$ in its lowest terms where

$$
B_{2}=\frac{1}{6}, \quad B_{4}=\frac{1}{30}, \quad B_{6}=\frac{1}{42}, \quad B_{8}=\frac{1}{30}, \quad B_{10}=\frac{5}{66}, \ldots
$$

are the Bernoulli numbers. Now by von Staudt's Theorem

$$
\delta_{\varpi-1} \equiv 0 \quad(\bmod \varpi),
$$

and also we have

$$
n^{\varpi}-n \equiv 0 \quad(\bmod \varpi) .
$$

And so the left hand side in (15.1) is of the form

$$
c^{\prime}+\varpi J
$$

where $c^{\prime}$ is a constant integer while that in (15.2) is of the form

$$
k+c P+\varpi J
$$


where $c$ and $k$ are constant integers.

It appears that $k$ can be taken as zero always. This involves the assertion that

$$
6 v_{\varpi+1}+(-1)^{(\varpi+1) / 2} \frac{\varpi+1}{2} \delta_{\varpi+1} \equiv 0 \quad(\bmod \varpi) .
$$

I have not yet proved this result but in every particular case this can actually found to be true. Thus (15.31) can be replaced by

$$
c P+\varpi J
$$

Now using (15.3), (15.5) and (9.8) we can show in particular cases that

$$
\left(Q^{3}-R^{2}\right)^{\left(\varpi^{2}-1\right) / 24}=q \frac{d J}{d q}+\left(Q^{3}-R^{2}\right) \sum k_{\ell, m} Q^{\ell} R^{m}+\varpi J
$$

where $k_{\ell, m}$ is a constant integer and the summation extends over all positive integral values of $\ell$ and $m$ (including zero) such that

$$
4 \ell+6 m=\varpi-13 .
$$

But it is obvious that

$$
(q ; q)_{\infty}^{\varpi^{2}-1}=\frac{\left(q^{\varpi^{2}} ; q^{\varpi^{2}}\right)_{\infty}}{(q ; q)_{\infty}}+\varpi J .
$$

It follows from (15.6) and (15.7) that

$$
q^{\left(\varpi^{2}-1\right) / 24} \frac{\left(q^{\varpi^{2}} ; q^{\varpi^{2}}\right)_{\infty}}{(q ; q)_{\infty}}=q \frac{d J}{d q}+\left(Q^{3}-R^{2}\right) \sum k_{\ell, m} Q^{\ell} R^{m}+\varpi J
$$

where the remark about the summation in (15.6) applies here also. From this we can always deduce in every particular case that

$$
\begin{aligned}
& \sum_{n=1}^{\infty} p\left(n \varpi+\varpi\left[\frac{\varpi}{24}\right]-\frac{\varpi^{2}-1}{24}\right) q^{n+[\varpi / 24]}\left(q^{\varpi} ; q^{\varpi}\right)_{\infty} \\
= & \left(Q^{3}-R^{2}\right)^{1+[\varpi / 24]} \sum k_{\ell, m} Q^{\ell} R^{m}+\varpi J
\end{aligned}
$$

where $k_{\ell, m}$ is a constant integer and the summation extends over all positive integral values of $\ell$ and $m$ (including zero) such that

$$
4 \ell+6 m=\varpi-13
$$

and $[t]$ denotes as usual the greatest integer in $t$.

Even though all these results are very difficult to prove in general they can be easily proved when $\varpi \leq 23$.

The condition (15.9a) should be replaced by

$$
4 \ell+6 m=\varpi-13-12\left[\frac{\varpi}{24}\right] .
$$


It is understandable that Ramanujan had missed the last term in (15.9b), since he likely had calculated examples only for $\varpi \leq 23$.

\section{Moduli 17, 19 and 23}

16. In these cases we can easily prove that

$$
\sum_{n=1}^{\infty} p(17 n-12) q^{n}\left(q^{17} ; q^{17}\right)_{\infty}=7 \sum_{n=1}^{\infty} \tau_{2}(n) q^{n}+17 J
$$

where

$$
\sum_{n=1}^{\infty} p(19 n-15) q^{n}\left(q^{19} ; q^{19}\right)_{\infty}=5 \sum_{n=1}^{\infty} \tau_{3}(n) q^{n}+19 J
$$

where

$$
\sum_{n=1}^{\infty} \tau_{3}(n) q^{n}=R q(q ; q)_{\infty}^{24}
$$

and

$$
\sum_{n=1}^{\infty} p(23 n-22) q^{n}\left(q^{23} ; q^{23}\right)_{\infty}=\sum_{n=1}^{\infty} \tau_{5}(n) q^{n}+23 J
$$

where

$$
\sum_{n=1}^{\infty} \tau_{5}(n) q^{n}=Q R q(q ; q)_{\infty}^{24}
$$

I have stated without proof in my previous paper ${ }^{18}$ that

$$
\left\{\begin{array}{l}
\sum_{n=1}^{\infty} \frac{\tau_{2}(n)}{n^{s}}=\prod_{p} \frac{1}{1-\tau_{2}(p) p^{-s}+p^{15-2 s}}, \\
\sum_{n=1}^{\infty} \frac{\tau_{3}(n)}{n^{s}}=\prod_{p} \frac{1}{1-\tau_{3}(p) p^{-s}+p^{17-2 s}}, \\
\sum_{n=1}^{\infty} \frac{\tau_{4}(n)}{n^{s}}=\prod_{p} \frac{1}{1-\tau_{4}(p) p^{-s}+p^{19-2 s}}, \\
\sum_{n=1}^{\infty} \frac{\tau_{5}(n)}{n^{s}}=\prod_{p} \frac{1}{1-\tau_{5}(p) p^{-s}+p^{21-2 s}}, \\
\sum_{n=1}^{\infty} \frac{\tau_{7}(n)}{n^{s}}=\prod_{p} \frac{1}{1-\tau_{7}(p) p^{-s}+p^{25-2 s}},
\end{array}\right.
$$

${ }^{18}$ See [80, eq. (108)]. 
where

$$
\sum_{n=1}^{\infty} \tau_{4}(n) q^{n}=Q^{2} q(q ; q)_{\infty}^{24}
$$

and

$$
\sum_{n=1}^{\infty} \tau_{7}(n) q^{n}=Q^{2} R q(q ; q)_{\infty}^{24}
$$

and $p$ assumes all prime values. All these seem to be capable of proof as the case of $\tau(n)$ by Mordell's method. ${ }^{19}$

Now using (16.4) we can deduce from (16.1), (16.2) and (16.3) that

$$
\begin{aligned}
& \sum_{n=1}^{\infty} p\left(n 17^{2}-12\right) q^{n}(q ; q)_{\infty}=c_{2} \sum_{n=1}^{\infty} \tau_{2}(n) q^{n}+17 J \\
& \sum_{n=1}^{\infty} p\left(n 19^{2}-15\right) q^{n}(q ; q)_{\infty}=c_{3} \sum_{n=1}^{\infty} \tau_{3}(n) q^{n}+19 J
\end{aligned}
$$

and

$$
\sum_{n=1}^{\infty} p\left(n 23^{2}-22\right) q^{n}(q ; q)_{\infty}=c_{5} \sum_{n=1}^{\infty} \tau_{5}(n) q^{n}+23 J
$$

where $c_{2}, c_{3}$ and $c_{5}$ are constants.

I have found that there are formulae quite analogous to those for modulus 13 even in these cases. I shall reserve the discussion of these as well as those for higher primes to another paper; but I shall consider in the II part of this paper the analogous formulae for the smaller primes 5, 7, and 11 .

The corresponding formulae for primes greater than 23 are not quite analogous. For instance in the cases of

we have

\section{$\underline{\text { moduli } 29 \text { and } 31}$}

$$
\sum_{n=1}^{\infty} p(29 n-6) q^{n+1}\left(q^{29} ; q^{29}\right)_{\infty}=8 \sum_{n=1}^{\infty} \Omega_{2}(n) q^{n}+29 J
$$

where

$$
\sum_{n=1}^{\infty} \Omega_{2}(n) q^{n}=Q q^{2}(q ; q)_{\infty}^{48}
$$

and

$$
\sum_{n=1}^{\infty} p(31 n-9) q^{n+1}\left(q^{31} ; q^{31}\right)_{\infty}=10 \sum_{n=1}^{\infty} \Omega_{3}(n) q^{n}+31 J
$$

where

$$
\sum_{n=1}^{\infty} \Omega_{3}(n) q^{n}=R q^{2}(q ; q)_{\infty}^{48}
$$

\footnotetext{
${ }^{19}$ loc. cit.
} 
The functions

$$
\sum_{n=1}^{\infty} \frac{\Omega_{2}(n)}{n^{s}}, \quad \sum_{n=1}^{\infty} \frac{\Omega_{3}(n)}{n^{s}}
$$

are obviously not capable of a single product as in (16.4); but they are, as a matter of fact, the differences of two such products.

17. I have not yet investigated the residues of $\tau(n)$ for other moduli besides what was stated before but the case 23 seems to be (comparatively) simple. For it appears that if

$$
\sum_{n=1}^{\infty} \lambda(n) q^{n}=q(q ; q)_{\infty}\left(q^{23} ; q^{23}\right)_{\infty}
$$

so that

$$
\tau(n)-\lambda(n) \equiv 0 \quad(\bmod 23)
$$

then

$$
\sum_{n=1}^{\infty} \frac{\lambda(n)}{n^{s}}=\frac{1}{1-23^{-s}} \prod_{1} \prod_{2} \prod_{3}
$$

where

$$
\prod_{1}=\prod_{p} \frac{1}{1-p^{-2 s}},
$$

$p$ assuming all prime values of the form ${ }^{20}$

$$
p \equiv 5,7,10,11,14,15,17,19,20,21,22 \quad(\bmod 23)
$$

and

$$
\prod_{2}=\prod_{p} \frac{1}{1+p^{-s}+p^{-2 s}}
$$

$p$ assuming all prime values of the form ${ }^{21}$

$$
p \equiv 1,2,3,4,6,8,9,12,13,16,18 \quad(\bmod 23)
$$

except of the form $23 a^{2}+b^{2}$, and

$$
\prod_{3}=\prod_{p} \frac{1}{\left(1-p^{-s}\right)^{2}}
$$

$p$ assuming all primes of the form $23 a^{2}+b^{2}$. Thus $\lambda(n)$ can be completely determined and consequently the residues of $\tau(n)$ for modulus 23 can be completely ascertained.

Suppose now that

$$
\begin{cases}t_{n}=0, & \tau(n) \equiv 0(\bmod 23) \\ t_{n}=1, & \tau(n) \neq 0(\bmod 23) .\end{cases}
$$

\footnotetext{
${ }^{20}$ This can be written as $p^{11} \equiv-1(\bmod 23)$.

${ }^{21}$ This can be written as $p^{11} \equiv 1(\bmod 23)$.
} 
Then it is easy to see from ( ) that

$$
\sum_{n=1}^{\infty} \frac{t_{n}}{n^{s}}=\prod_{1} \prod_{2} \prod_{3}
$$

where

$$
\prod_{1}=\prod_{p} \frac{1}{1-p^{-2 s}}
$$

$p$ assuming all primes of the form (17.3),

$$
\prod_{2}=\prod_{p} \frac{1+p^{-s}}{1-p^{-3 s}}
$$

$p$ assuming all primes of the form (17.4) except those of the form $23 a^{2}+b^{2}$, and

$$
\prod_{3}=\prod_{p} \frac{1-p^{-22 s}}{\left(1-p^{-s}\right)\left(1-p^{-23 s}\right)}
$$

$p$ assuming all primes of the form $23 a^{2}+b^{2}$.

It is easy to prove from (17.6) by quite elementary methods that

$$
\sum_{k=1}^{n} t_{k}=o(n)
$$

and by transcendental methods that

$$
\sum_{k=1}^{n} t_{k}=C \int_{1}^{n} \frac{d x}{(\log x)^{1 / 2}}+O\left(\frac{n}{(\log n)^{r}}\right),
$$

where $r$ is any positive number and

$$
\begin{aligned}
C= & \frac{66^{1 / 2}}{23^{3 / 4}} \frac{1-2^{-2}}{1-2^{-3}} \frac{1-3^{-2}}{1-3^{-3}} \frac{1-13^{-2}}{1-13^{-3}} \frac{1-29^{-2}}{1-29^{-3}} \cdots \\
& \times \frac{1}{\left\{\left(1-5^{-2}\right)\left(1-7^{-2}\right)\left(1-11^{-2}\right)\left(1-17^{-2}\right) \cdots\right\}^{1 / 2}} \\
& \times \frac{1-59^{-22}}{1-59^{-23}} \frac{1-101^{-22}}{1-101^{-23}} \frac{1-167^{-22}}{1-167^{-23}} \cdots,
\end{aligned}
$$

$2,3,13, \ldots$ being primes of the form (17.4) except those of the form $23 a^{2}+b^{2}$, and $5,7,11,17, \ldots$ being primes of the form $(17.3)$ and $59,101,167, \ldots$ are those of the form $23 a^{2}+b^{2}$. Thus we see that $\tau(n)$ is almost always divisible by 23 .

We have also shown that among the values of $\tau(n)$, multiples of 3,7 and 23 are more or less equally numerous while the multiples of 5 are less numerous than these and multiples of 2 are the most numerous.

Since

$$
\begin{aligned}
\left(1-p^{-s}\right)\left(1-p^{11-s}\right) & =\left(1-p^{-2 s}\right)-\left(p^{11}+1\right)\left(p^{-s}-p^{-2 s}\right) \\
& =\left(1-p^{-s}\right)^{2}-\left(p^{11}-1\right)\left(p^{-s}-p^{-2 s}\right)
\end{aligned}
$$


it is easy to see from (17.2) and (12.7) that if the prime divisors of $n$ are of the form (17.3) or of the form $23 a^{2}+b^{222}$ then

$$
\tau(n)-\sigma_{11}(n) \equiv 0 \quad(\bmod 15893),
$$

15893 being $23 \cdot 691$. If, in addition to the restrictions on the values of $n$ in (17.9), we impose the restriction that $n$ is odd also then if follows from (12.9) that

$$
\tau(n)-\sigma_{11}(n) \equiv 0 \quad(\bmod 4068608)
$$

4068608 being $23 \cdot 256 \cdot 691$.

\section{Modulus 121}

18. The case of modulus $\varpi^{2}$ seems to be much more complicated than the case of modulus $\varpi$ even though the method is practically the same as may be seen from the case of modulus 49. I shall now consider the case of modulus 121.

It is easy to show by using (9.2) that

$$
\left(Q^{3}-R^{2}\right)^{5}=P\left(Q^{3}-3 R^{2}\right)\left(3 P^{3}-P Q+4 R\right)+4 Q R\left(4 P^{3} Q-3 P^{2} R+2 Q R\right)
$$

$$
-26 P^{5}+23 P^{3} Q+16 P^{2} R-22 P Q^{2}+9 Q R+121 J \text {. }
$$

From this we can deduce that

$$
\begin{aligned}
q^{5} \frac{\left(q^{11} ; q^{11}\right)_{\infty}^{11}}{(q ; q)_{\infty}}= & \sum_{n=1}^{\infty}\left[n^{4}\left\{a_{1} \sigma_{1}(n)+b_{1} \sigma_{11}(n)\right\}+n^{3}\left\{a_{2} \sigma_{3}(n)+b_{2} \sigma_{13}(n)\right\}\right. \\
& +n^{2}\left\{a_{3} \sigma_{5}(n)+b_{3} \sigma_{15}(n)\right\}+n\left\{a_{4} \sigma_{7}(n)+b_{4} \sigma_{17}(n)\right\} \\
& \left.+c_{1} n^{2} \tau_{2}(n)+c_{2} n \tau_{3}(n)+c_{3} \tau_{4}(n)\right] q^{n}+121 J
\end{aligned}
$$

where the $a$ 's, $b$ 's and $c$ 's are constant integers and $\tau_{2}(n), \tau_{3}(n)$ and $\tau_{4}(n)$ are the same as in (16.4). But it is easy to show that

$$
\left\{\begin{array}{l}
\tau_{2}(n)-n \sigma_{3}(n), \\
\tau_{3}(n)-n \sigma_{5}(n), \quad \equiv 0(\bmod 11) . \\
\tau_{4}(n)-n \sigma_{7}(n),
\end{array}\right.
$$

It is easy to see from (16.4) that

$$
\tau_{4}(11 n)-\tau_{4}(11) \tau_{4}(n) \equiv 0 \quad(\bmod 121),
$$

and by actual calculation we find that

$$
\tau_{4}(11) \equiv 0 \quad(\bmod 11)
$$

It is also obvious that

$$
\sigma_{17}(n)-\sigma_{7}(n) \equiv 0 \quad(\bmod 11) .
$$

\footnotetext{
${ }^{22}$ Some may be of one form and some may be of the other form.
} 
Now remembering (18.3)-(18.6) and picking out the terms $q^{11}, q^{22}, q^{33}, \ldots$ in ( ) we obtain

$$
\sum_{n=1}^{\infty} p(11 n-5) q^{n}\left(q^{11} ; q^{11}\right)_{\infty}=11 \sum_{n=1}^{\infty} n \sigma_{7}(n) q^{n}+121 J
$$

It follows from this that

$$
p(121 n-5) \equiv 0 \quad(\bmod 121)
$$

and

$$
\begin{gathered}
p(11 n-5)-p(11 n-126)-p(11 n-247) \\
+p(11 n-610)+\cdots-11 n \sigma_{7}(n) \equiv 0(\bmod 121) .
\end{gathered}
$$

19. In concluding the first part of this paper I shall consider the numbers which are the divisors of $\tau(n)$ for almost all values of $n$.

Suppose that $\varpi_{1}, \varpi_{2}, \varpi_{3}, \ldots$ are an infinity of primes such that

$$
\sum_{n=1}^{\infty} \frac{1}{\varpi_{n}}
$$

is a divergent series and also suppose that $a_{2}, a_{3}, a_{5}, a_{7}, \ldots$ assume some or all of the positive integers (including zero) but that $a_{\varpi_{1}}, a_{\varpi_{2}}, a_{\varpi_{3}}, \ldots$ never assume the value unity. Then it is easy to show that the number of numbers of the form

$$
2^{a_{2}} \cdot 3^{a_{3}} \cdot 5^{a_{5}} \cdot 7^{a_{7}} \cdots
$$

not exceeding $n$ is of the form

$$
o(n) .
$$

In particular if $a_{\varpi}$ never assumes the value unity for all prime values of $\varpi$ of the form

$$
\varpi \equiv c \quad(\bmod k),
$$

where $c$ and $k$ are any two integers which are prime to each other, then the number of numbers of the form (19.2) is of the form

$$
o(n)
$$

and more accurately is of the form

$$
O\left(\frac{n}{(\log n)^{1 /(k-1)}}\right)
$$

where $k$ is the same as in (19.4). 
Thus for example if $s$ be an odd positive integer, the number of values of $n$ not exceeding $n$ for which $\sigma_{s}(n)$ is not divisible by $k$, where $k$ is any positive integer, is of the form

$$
o(n)
$$

and more accurately is of the form

$$
O\left(\frac{n}{(\log n)^{1 /(k-1)}}\right) .
$$

For if $n$ be written in the form

$$
2^{a_{2}} \cdot 3^{a_{3}} \cdot 5^{a_{5}} \cdot 7^{a_{7}} \cdots
$$

then we have

$$
\sigma_{s}(n)=\prod_{p} \frac{p^{s\left(1+a_{p}\right)}-1}{p^{s}-1}, \quad p=2,3,5,7,11, \ldots
$$

Since $s$ is odd, $\sigma_{s}(n)$ is divisible by $k$ at any rate when $a_{p}=1$ for all values of $p$ of the form

$$
p \equiv-1 \quad(\bmod k)
$$

and hence the results stated follow. Thus we see that, if $s$ is odd, $\sigma_{s}(n)$ is divisible by any given integer for almost all values of $n$.

It follows from all these and the formulae in Sections 4, 8, 12, and 17, that

$$
\tau(n) \equiv 0 \quad\left(\bmod 2^{5} \cdot 3^{3} \cdot 5^{2} \cdot 7^{2} \cdot 23 \cdot 691\right)
$$

for almost all values of $n$.

It appears that $\tau(n)$ is almost always divisible by any power of 2,3 , and 5 . It also appears from Section 9 that there are reasons to suppose that $\tau(n)$ is almost always divisible by 11 also. But I have no evidence at present to say anything about the other powers of 7 and other primes one way or the other.

Among the values of $\tau(n)$ multiples of $2,3,5,7$ and 23 are very numerous from the beginning but multiples of 691 begin at a very late stage. For instance $\tau(n)$ is divisible by 23 for 132 values of $n$ not exceeding 200 while the first value of $n$ for which $\tau(n)$ is divisible by 691 is 1381 and this is the only such value of $n$ among the first 5000 values.

\section{II}

\section{Moduli 5 and 25}

20. In this second part we shall use $J_{1}, J_{2}, J_{3}$ and $G_{1}, G_{2}, G_{3}$ to denote functions of $q$ with integral powers of $q$ as well as integral coefficients. These are the same functions in the same section unlike $J$. We shall also use $J$ in the same sense as in the first part.

We start with Euler's identities

$$
(q ; q)_{\infty}=\sum_{n=-\infty}^{\infty}(-1)^{n} q^{n(3 n-1) / 2}
$$


and Jacobi's identity

$$
(q ; q)_{\infty}^{3}=\sum_{n=0}^{\infty}(-1)^{n}(2 n+1) q^{n(n+1) / 2} .
$$

It is easy to see from (20.1) that

$$
\frac{\left(q^{1 / 5} ; q^{1 / 5}\right)_{\infty}}{\left(q^{5} ; q^{5}\right)_{\infty}}=J_{1}-q^{1 / 5}+q^{2 / 5} J_{2}
$$

Now cubing both sides we obtain

$$
\begin{aligned}
& \frac{\sum_{n=0}^{\infty}(-1)^{n}(2 n+1) q^{n(n+1) / 10}}{\sum_{n=0}^{\infty}(-1)^{n}(2 n+1) q^{5 n(n+1) / 2}}=\left(J_{1}^{3}-3 J_{2}^{2} q\right)-q^{1 / 5}\left(3 J_{1}^{2}-J_{2}^{3} q\right)+3 J_{1} q^{2 / 5}\left(1+J_{1} J_{2}\right) \\
& (20.3) \\
& -q^{3 / 5}\left(1+6 J_{1} J_{2}\right)+3 J_{2} q^{4 / 5}\left(1+J_{1} J_{2}\right) .
\end{aligned}
$$

But it is easy to see that

$$
\frac{\sum_{n=0}^{\infty}(-1)^{n}(2 n+1) q^{n(n+1) / 10}}{\sum_{n=0}^{\infty}(-1)^{n}(2 n+1) q^{5 n(n+1) / 2}}=G_{1}+q^{1 / 5} G_{2}+5 q^{3 / 5} .
$$

Hence

$$
J_{1}\left(1+J_{1} J_{2}\right)=0, \quad 1+6 J_{1} J_{2}=-5, \quad J_{2}\left(1+J_{1} J_{2}\right)=0 .
$$

These three equations give one and the same relation between $J_{1}$ and $J_{2}$, viz.

$$
J_{1} J_{2}=-1 \text {. }
$$

Using this we obtain

$$
\frac{\left(q^{5} ; q^{5}\right)_{\infty}}{\left(q^{1 / 5} ; q^{1 / 5}\right)_{\infty}}=\frac{1}{J_{1}-q^{1 / 5}+q^{2 / 5} J_{2}}
$$

$$
=\frac{\left(J_{1}^{4}+3 J_{2} q\right)+q^{1 / 5}\left(J_{1}^{3}+2 J_{2}^{2} q\right)+q^{2 / 5}\left(2 J_{1}^{2}+J_{2}^{3} q\right)+q^{3 / 5}\left(3 J_{1}+J_{2}^{4} q\right)+5 q^{4 / 5}}{J_{1}^{5}-11 q+q^{2} J_{2}^{5}}
$$

by rationalizing the denominator $J_{1}-q^{1 / 5}+q^{2 / 5} J_{2}$. It follows from (20.5) that

$$
\sum_{n=0}^{\infty} p(5 n+4) q^{n}\left(q^{5} ; q^{5}\right)_{\infty}=\frac{5}{J_{1}^{5}-11 q+q^{2} J_{2}^{5}} .
$$

But we see from (20.2) that

$$
\frac{\left(\omega q^{1 / 5} ; \omega q^{1 / 5}\right)_{\infty}}{\left(q^{5} ; q^{5}\right)_{\infty}}=J_{1}-\omega q^{1 / 5}+\omega^{2} q^{2 / 5} J_{2}
$$

where $\omega^{5}=1$. Now writing the five values of $\omega$ in (20.21) and multiplying them together we obtain

$$
\frac{(q ; q)_{\infty}^{6}}{\left(q^{5} ; q^{5}\right)_{\infty}^{6}}=J_{1}^{5}-11 q+q^{2} J_{2}^{5}
$$


It follows from this and (20.6) that

$$
\sum_{n=0}^{\infty} p(5 n+4) q^{n}=5 \frac{\left(q^{5} ; q^{5}\right)_{\infty}^{5}}{(q ; q)_{\infty}^{6}}
$$

It follows that

$$
p(5 n-1) \equiv 0 \quad(\bmod 5)
$$

Again the right hand side in (20.8) is of the form

$$
5 \frac{\left(q^{5} ; q^{5}\right)_{\infty}^{4}}{(q ; q)_{\infty}}+25 J
$$

It follows from this and (20.81) that the coefficients of $q^{4}, q^{9}, q^{14}, \ldots$ in this are all multiples of 25 and consequently the coefficient of $q^{5 n-1}$ in the left hand side of (20.8) is a multiple of 25 . In other words

$$
p(25 n-1) \equiv 0 \quad(\bmod 25)
$$

It follows also from (20.8) that

$$
\sum_{n=0}^{\infty} p(5 n+4) q^{n}=5(q ; q)_{\infty}^{19}+125 J
$$

\section{Modulus 125}

21. Changing $q$ to $q^{1 / 5}$ in (20.8) and arguing as before, using (20.5) and (20.7) we find that

$$
\begin{aligned}
\sum_{n=0}^{\infty} p(25 n+24) q^{n}= & 5^{2} \cdot 63 \frac{\left(q^{5} ; q^{5}\right)_{\infty}^{6}}{(q ; q)_{\infty}^{7}}+5^{5} \cdot 52 q \frac{\left(q^{5} ; q^{5}\right)_{\infty}^{12}}{(q ; q)_{\infty}^{13}}+5^{7} \cdot 63 q^{2} \frac{\left(q^{5} ; q^{5}\right)_{\infty}^{18}}{(q ; q)_{\infty}^{19}} \\
& +5^{10} \cdot 6 q^{3} \frac{\left(q^{5} ; q^{5}\right)_{\infty}^{24}}{(q ; q)_{\infty}^{25}}+5^{12} q^{4} \frac{\left(q^{5} ; q^{5}\right)_{\infty}^{30}}{(q ; q)_{\infty}^{31}} .
\end{aligned}
$$

Now

$$
\frac{\left(q^{5} ; q^{5}\right)_{\infty}^{6}}{(q ; q)_{\infty}^{7}}=\sum_{n=0}^{\infty}(-1)^{n}(2 n+1) q^{n(n+1) / 2}\left(q^{5} ; q^{5}\right)_{\infty}^{4}+5 J \quad \text { etc. }
$$

and the coefficients of $q^{5 n-1}, q^{5 n-2}, q^{5 n-3}$ in $\sum_{n=0}^{\infty}(-1)^{n}(2 n+1) q^{n(n+1) / 2}$ are easily seen to be zero or multiples of 5 . It follows that the coefficients of $q^{5 n-1}, q^{5 n-2}, q^{5 n-3}$ in the left hand side of (21.1) are multiples of 125. In other words

$$
\left\{\begin{array}{l}
p(125 n-1) \\
p(125 n-26) \equiv 0(\bmod 125) \\
p(125 n-51)
\end{array}\right.
$$


It is also easy to see from (21.1) that

$$
\sum_{n=0}^{\infty} p(25 n+24) q^{n}=75(q ; q)_{\infty}^{23}+125 J
$$

The right hand side in (21.4) can be written in the form

$$
75 \frac{(q ; q)_{\infty}^{48}}{\left(q^{25} ; q^{25}\right)_{\infty}}+125 J
$$

But it is easy to show that

$$
\left(Q^{3}-R^{2}\right)^{2}=-2 \sum_{n=1}^{\infty}\left(n^{3}-n\right) \sigma_{1}(n) q^{n}+5 J
$$

[To prove (21.6), we need Ramanujan's formula [80, Table III], [85, p. 142]

$$
6912 \sum_{n=1}^{\infty} n^{3} \sigma_{1}(n) q^{n}=6 P^{2} Q-8 P R+3 Q^{2}-P^{4}
$$

Using this formula together with (1.4) and (1.2), we can readily prove that

$$
2 \sum_{n=1}^{\infty}\left(n^{3}-n\right) \sigma_{1}(n) q^{n}=-1+2 P^{2}-P^{4}+5 J
$$

On the other hand, from (1.2) and (1.3),

$$
\left(Q^{3}-R^{2}\right)^{2}=1-2 P^{2}+P^{4}+5 J .
$$

The last two equalities yield (21.6).] It follows that

$$
\sum_{n=0}^{\infty} p(25 n+24) q^{n+2}\left(q^{25} ; q^{25}\right)_{\infty}=25 \sum_{n=1}^{\infty}\left(n^{3}-n\right) \sigma_{1}(n) q^{n}+125 J
$$

In other words

$$
\begin{aligned}
& p(25 n-26)-p(25 n-651)-p(25 n-1276) \\
& +p(25 n-3151)+\cdots-25\left(n^{3}-n\right) \sigma_{1}(n) \equiv 0(\bmod 125) .
\end{aligned}
$$

$p(199)$ is the coefficient of $q^{7}$ in (21.2).

$p(199)=5^{2} \cdot 63 \cdot 12195+5^{2} \cdot 52 \cdot 60541+5^{7} \cdot 63 \cdot 66862+5^{10} \cdot 6 \cdot 29575+5^{12} \cdot 6448$ $=3646072432125$. 
22. Changing again $q$ to $q^{1 / 5}$ in (21.1) and arguing as before using (20.5) and (20.7) we can show that

$$
\sum_{n=0}^{\infty} p(125 n+99) q^{n}=\sum_{r=1}^{25} a_{r} \frac{\left(q^{5} ; q^{5}\right)_{\infty}^{6 r-1}}{(q ; q)_{\infty}^{6 r}}
$$

where the $a$ 's are positive integers such that $a_{1}=p(99)=5^{3} \cdot 1353839$ and $a_{2}, a_{3}, a_{4}, \ldots$ contain higher powers of 5 than $a_{1}$ as factors. It is easy to see from this that

$$
\sum_{n=0}^{\infty} p(125 n+99) q^{n}=4 \cdot 5^{3}(q ; q)_{\infty}^{19}+5^{4} J
$$

In this way arguing as before, we can show that if $\lambda$ be any positive odd integer, then

$$
\sum_{n=0}^{\infty} p\left(\frac{19 \cdot 5^{\lambda}+1}{24}+5^{\lambda} n\right) q^{n}=\sum_{\nu=1}^{5^{\lambda-1}} a_{\nu} \frac{\left(q^{5} ; q^{5}\right)_{\infty}^{6 \nu-1}}{(q ; q)_{\infty}^{6 \nu}}
$$

where the $a$ 's are positive integers such that $a_{2}, a_{3}, a_{4}, \ldots$ contain higher powers of 5 than $a_{1}$ as factors; and if $\lambda$ be a positive even integer then

$$
\sum_{n=0}^{\infty} p\left(\frac{23 \cdot 5^{\lambda}+1}{24}+5^{\lambda} n\right) q^{n}=\sum_{\nu=1}^{5^{\lambda-1}} a_{\nu} \frac{\left(q^{5} ; q^{5}\right)_{\infty}^{6 \nu}}{(q ; q)_{\infty}^{6 \nu+1}}
$$

where the $a$ 's have the same properties as before. We deduce from (22.3) and (22.4) that if $\lambda$ is a positive odd integer then

$$
\sum_{n=0}^{\infty} p\left(\frac{19 \cdot 5^{\lambda}+1}{24}+5^{\lambda} n\right) q^{n}=c_{\lambda} \cdot 5^{\lambda}(q ; q)_{\infty}^{19}+5^{\lambda+1} J
$$

and if $\lambda$ is a positive even integer then

$$
\sum_{n=0}^{\infty} p\left(\frac{23 \cdot 5^{\lambda}+1}{24}+5^{\lambda} n\right) q^{n}=c_{\lambda} \cdot 5^{\lambda}(q ; q)_{\infty}^{23}+5^{\lambda+1} J
$$

where $c_{\lambda}$ in both cases is a constant.

We easily deduce from these that if $\lambda$ is an odd integer greater than 1 , then

$$
\left\{\begin{array}{l}
p\left(5^{\lambda} n-\frac{5^{\lambda-1}-1}{24}\right) \\
p\left(5^{\lambda} n-\frac{5^{\lambda+1}-1}{24}\right)^{23} \equiv 0\left(\bmod 5^{\lambda}\right) \\
p\left(5^{\lambda} n-\frac{49 \cdot 5^{\lambda-1}-1}{24}\right)
\end{array}\right.
$$


and if $\lambda$ is a positive even integer, then

$$
p\left(5^{\lambda} n-\frac{5^{\lambda}-1}{24}\right) \equiv 0 \quad\left(\bmod 5^{\lambda}\right) .
$$

23. We have seen that we can take $c_{1}=1, c_{2}=-2, c_{3}=4$ in (22.5) and (22.6). It appears from Section 22 that $c_{\lambda}$ may probably be some simple function such as $(-2)^{\lambda}$. If we calculate a few more values of $c_{\lambda}$, we can definitely know what it is. Then we can make use of the formulae (22.5) and (22.6) to determine completely the residues of

$$
p\left(5^{\lambda} n-\frac{5^{\lambda+1}-1}{24}\right)
$$

for odd values of $\lambda$ and those of

$$
p\left(5^{\lambda} n-\frac{5^{\lambda}-1}{24}\right)
$$

for even values of $\lambda$ for modulus $5^{\lambda+1}$. [To determine these residues, we need the expansions

$$
\begin{aligned}
(q ; q)_{\infty}^{19}= & 1-19 q+152 q^{2}-627 q^{3}+1140 q^{4}+988 q^{5}-9063 q^{6} \\
& +14212 q^{7}+7410 q^{8}-44270 q^{9}+22781 q^{10}+38114 q^{11} \\
& +36176 q^{12}-137256 q^{13}-154850 q^{14}+480605 q^{15}+\cdots
\end{aligned}
$$

and

$$
\begin{aligned}
(q ; q)_{\infty}^{23}= & 1-23 q+230 q^{2}-1265 q^{3}+3795 q^{4}-3519 q^{5}-16445 q^{6} \\
& +64285 q^{7}-64515 q^{8}-120175 q^{9}+354706 q^{10}-123763 q^{11} \\
& -407560 q^{12}-48530 q^{13}+817190 q^{14}+1464341 q^{15}+\cdots
\end{aligned}
$$

in, respectively, (22.5) and (22.6).] Thus for instance if follows immediately from (22.5) and (22.6) that if $\lambda$ is an odd integer then

$$
\begin{array}{ll}
p\left(5^{\lambda}-\frac{5^{\lambda+1}-1}{24}\right)-5^{\lambda} c_{\lambda}, & p\left(2 \cdot 5^{\lambda}-\frac{5^{\lambda+1}-1}{24}\right)-5^{\lambda} c_{\lambda}, \\
p\left(3 \cdot 5^{\lambda}-\frac{5^{\lambda+1}-1}{24}\right)-2 \cdot 5^{\lambda} c_{\lambda}, & p\left(4 \cdot 5^{\lambda}-\frac{5^{\lambda+1}-1}{24}\right)+2 \cdot 5^{\lambda} c_{\lambda}, \\
p\left(5 \cdot 5^{\lambda}-\frac{5^{\lambda+1}-1}{24}\right), & p\left(6 \cdot 5^{\lambda}-\frac{5^{\lambda+1}-1}{24}\right)-2 \cdot 5^{\lambda} c_{\lambda}, \\
p\left(7 \cdot 5^{\lambda}-\frac{5^{\lambda+1}-1}{24}\right)-2 \cdot 5^{\lambda} c_{\lambda}, & p\left(8 \cdot 5^{\lambda}-\frac{5^{\lambda+1}-1}{24}\right)-2 \cdot 5^{\lambda} c_{\lambda}, \\
p\left(9 \cdot 5^{\lambda}-\frac{5^{\lambda+1}-1}{24}\right), & p\left(10 \cdot 5^{\lambda}-\frac{5^{\lambda+1}-1}{24}\right), \\
p\left(11 \cdot 5^{\lambda}-\frac{5^{\lambda+1}-1}{24}\right)-5^{\lambda} c_{\lambda}, & p\left(12 \cdot 5^{\lambda}-\frac{5^{\lambda+1}-1}{24}\right)+5^{\lambda} c_{\lambda}, \\
p\left(13 \cdot 5^{\lambda}-\frac{5^{\lambda+1}-1}{24}\right)-5^{\lambda} c_{\lambda}, & p\left(14 \cdot 5^{\lambda}-\frac{5^{\lambda+1}-1}{24}\right)+5^{\lambda} c_{\lambda}, \\
p\left(15 \cdot 5^{\lambda}-\frac{5^{\lambda+1}-1}{24}\right), & p\left(16 \cdot 5^{\lambda}-\frac{5^{\lambda+1}-1}{24}\right),
\end{array}
$$

${ }^{23} \lambda$ may also be 1 in this formula. 
and so on are all multiples of $5^{\lambda+1}$; and if $\lambda$ is an even integer, then

$$
\begin{array}{ll}
p\left(5^{\lambda}-\frac{5^{\lambda}-1}{24}\right)-5^{\lambda} c_{\lambda}, & p\left(2 \cdot 5^{\lambda}-\frac{5^{\lambda}-1}{24}\right)-2 \cdot 5^{\lambda} c_{\lambda}, \\
p\left(3 \cdot 5^{\lambda}-\frac{5^{\lambda}-1}{24}\right), & p\left(4 \cdot 5^{\lambda}-\frac{5^{\lambda}-1}{24}\right), \\
p\left(5 \cdot 5^{\lambda}-\frac{5^{\lambda}-1}{24}\right), & p\left(6 \cdot 5^{\lambda}-\frac{5^{\lambda}-1}{24}\right)-5^{\lambda} c_{\lambda}, \\
p\left(7 \cdot 5^{\lambda}-\frac{5^{\lambda}-1}{24}\right), & p\left(8 \cdot 5^{\lambda}-\frac{5^{\lambda}-1}{24}\right), \\
p\left(9 \cdot 5^{\lambda}-\frac{5^{\lambda}-1}{24}\right), & p\left(10 \cdot 5^{\lambda}-\frac{5^{\lambda}-1}{24}\right), \\
p\left(11 \cdot 5^{\lambda}-\frac{5^{\lambda}-1}{24}\right)-5^{\lambda} c_{\lambda}, & p\left(12 \cdot 5^{\lambda}-\frac{5^{\lambda}-1}{24}\right)-2 \cdot 5^{\lambda} c_{\lambda}, \\
p\left(13 \cdot 5^{\lambda}-\frac{5^{\lambda}-1}{24}\right), & p\left(14 \cdot 5^{\lambda}-\frac{5^{\lambda}-1}{24}\right), \\
p\left(15 \cdot 5^{\lambda}-\frac{5^{\lambda}-1}{24}\right), & p\left(16 \cdot 5^{\lambda}-\frac{5^{\lambda}-1}{24}\right)-5^{\lambda} c_{\lambda},
\end{array}
$$

and so on are all multiples of $5^{\lambda+1}$.

\section{Moduli 7 and 49}

24. It is easy to see from (20.1) that

$$
\frac{\left(q^{1 / 7} ; q^{1 / 7}\right)_{\infty}}{\left(q^{7} ; q^{7}\right)_{\infty}}=J_{1}+q^{1 / 7} J_{2}-q^{2 / 7}+q^{5 / 7} J_{3}
$$

Now cubing both sides we obtain

$$
\begin{aligned}
& \frac{\sum_{n=0}^{\infty}(-1)^{n}(2 n+1) q^{n(n+1) / 14}}{\sum_{n=0}^{\infty}(-1)^{n}(2 n+1) q^{7 n(n+1) / 2}} \\
= & \left(J_{1}^{3}+3 J_{2}^{2} J_{3} q-6 J_{1} J_{3} q\right)+q^{1 / 7}\left(3 J_{1}^{2} J_{2}-6 J_{2} J_{3} q+J_{3}^{2} q^{2}\right)+3 q^{2 / 7}\left(J_{1} J_{2}^{2}-J_{1}^{2}+J_{3} q\right) \\
& +q^{3 / 7}\left(J_{2}^{3}-6 J_{1} J_{2}+3 J_{1} J_{3}^{2} q\right)+3 q^{4 / 7}\left(J_{1}-J_{2}^{2}+J_{2} J_{3}^{2} q\right) \\
& +3 q^{5 / 7}\left(J_{2}+J_{1}^{2} J_{3}-J_{3}^{2} q\right)+q^{6 / 7}\left(6 J_{1} J_{2} J_{3}-1\right) .
\end{aligned}
$$

But it is easy to see that

$$
\frac{\sum_{n=0}^{\infty}(-1)^{n}(2 n+1) q^{n(n+1) / 14}}{\sum_{n=0}^{\infty}(-1)^{n}(2 n+1) q^{7 n(n+1) / 2}}=G_{1}+q^{1 / 7} G_{2}+q^{3 / 7} G_{3}-7 q^{6 / 7} .
$$

Hence

$$
\left\{\begin{aligned}
J_{1} J_{2}^{2}-J_{1}^{2}+J_{3} q & =0 \\
J_{1}-J_{2}^{2}+J_{2} J_{3}^{2} q & =0 \\
J_{2}+J_{1}^{2} J_{3}-J_{3}^{2} q & =0 \\
6 J_{1} J_{2} J_{3}-1 & =-7 .
\end{aligned}\right.
$$


All these four equations give the two independent relations

$$
J_{1} J_{2} J_{3}=-1, \quad \frac{J_{1}^{2}}{J_{3}}+\frac{J_{2}}{J_{3}^{2}}=q .
$$

Now write (24.1) in the form

$$
\frac{\left(\omega q^{1 / 7} ; \omega q^{1 / 7}\right)_{\infty}}{\left(q^{7} ; q^{7}\right)_{\infty}}=J_{1}+\omega q^{1 / 7} J_{2}-\omega^{2} q^{2 / 7}+\omega^{5} q^{5 / 7} J_{3}
$$

where $\omega^{7}=1$. Again writing the seven values of $\omega$ in (24.3) and multiplying them together and using (24.2a) we can show that

$$
\begin{aligned}
J_{1}^{7}+J_{2}^{7} q+J_{3}^{7} q^{5} & =\frac{(q ; q)_{\infty}^{8}}{\left(q^{7} ; q^{7}\right)_{\infty}^{8}}+14 q \frac{(q ; q)_{\infty}^{4}}{\left(q^{7} ; q^{7}\right)_{\infty}^{4}}+57 q^{3}, \\
J_{1}^{3} J_{2}+J_{2}^{3} J_{3} q+J_{3}^{3} J_{1} q^{2} & =-\frac{(q ; q)_{\infty}^{4}}{\left(q^{7} ; q^{7}\right)_{\infty}^{4}}-8 q \\
J_{1}^{2} J_{2}^{3}+J_{3}^{2} J_{1}^{3} q+J_{2}^{2} J_{3}^{3} q^{2} & =-\frac{(q ; q)_{\infty}^{4}}{\left(q^{7} ; q^{7}\right)_{\infty}^{4}}-5 q .
\end{aligned}
$$

Again taking the reciprocals of both sides in (24.1) and rationalizing the denominator by using as in Section 20, we can show that

$$
\begin{gathered}
\sum_{n=0}^{\infty} p(7 n+5) q^{n}=7 \frac{\left(q^{7} ; q^{7}\right)_{\infty}^{3}}{(q ; q)_{\infty}^{4}}+49 q \frac{\left(q^{7} ; q^{7}\right)_{\infty}^{7}}{(q ; q)_{\infty}^{8}} \\
7^{2} \cdot 2546,7^{4} \cdot 48 \cdot 934,7^{5} \cdot 1418989,7^{8} \cdot 335400 \\
\left\{p(47) q^{3}+\cdots\right\}\left(q^{49} ; q^{49}\right)_{\infty}=7 \sum_{n=1}^{\infty}\left\{22 n^{4} \sigma_{0}(n)-21 n^{2} \sigma_{1}(n)-\tau(n)\right\} q^{n}+7^{3} J
\end{gathered}
$$

\section{COMMENTARY}

0. The designation, Section 0, for the first batch of Ramanujan's insertions is due to the present authors.

K. G. Ramanathan [78] also observed that $\tau(n)$ is even unless $n$ is an odd square.

The congruences $\tau(7 n-r) \equiv 0(\bmod 7), r=0,1,2,4$, were evidently first proved by J. R. Wilton [111]. G. H. Hardy, in his book Ramanujan [50, pp. 165-166] also gives a proof, as does Ramanathan [79].

The congruences $\tau(23 n-r) \equiv 0(\bmod 23)$, where $r$ is a quadratic residue modulo 23, were also first established by Wilton [111].

1. Without the insertions, the beginning of the paper actually begins with the definitions of the Eisenstein series $P, Q$, and $R$, which are denoted by $L, M$, and $N$, respectively, in Ramanujan's notebooks [84]. Since the remainder of this section was extracted for [83] with additional details supplied by Hardy, we have not added more details here. However, it seems appropriate here to provide an introduction 
to congruences for the partition function in arithmetic progressions, since a large portion of the manuscript focuses on this topic.

In this manuscript Ramanujan proves his well known congruences for $p(n)$, namely,

$$
\begin{aligned}
p(5 n+4) & \equiv 0(\bmod 5), \\
p(7 n+5) & \equiv 0(\bmod 7), \\
p(11 n+6) & \equiv 0(\bmod 11)
\end{aligned}
$$

These congruences are the first cases of the infinite families,

$$
\begin{aligned}
p\left(5^{k} n+\delta_{5, k}\right) & \equiv 0\left(\bmod 5^{k}\right), \\
p\left(7^{k} n+\delta_{7, k}\right) & \equiv 0\left(\bmod 7^{[k / 2]+1}\right), \\
p\left(11^{k} n+\delta_{11, k}\right) & \equiv 0\left(\bmod 11^{k}\right),
\end{aligned}
$$

where $\delta_{p, k}:=1 / 24\left(\bmod p^{k}\right)$. The literature on these congruences is extensive, and there are now many proofs and approaches to them, e.g., [3], [5], [30], [40], [41], [43], [44], [45], [52], [53], [54], [56], [67], [71], [76], and [108].

These congruences are indeed surprising for they appear to be examples of a very rare and isolated phenomenon. In fact, Ramanujan [82], [85, p. 230] remarked that "It appears that there are no equally simple properties for any moduli involving primes other than these three."

In view of Ramanujan's claim, it is natural to ask about the frequency of congruences for $p(n)$ and the possibility of finding new ones. In this direction, the second author has made some progress [70], [72] towards quantifying the rarity of such congruences, and A. O. L. Atkin and J. N. O'Brien [6], [7] have found other congruences for $p(n)$. For instance, Atkin has proved that

$$
p(17303 n+237) \equiv 0 \quad(\bmod 13) .
$$

It is reasonable to conclude that such congruences are quite rare, but not so rare that one cannot find infinitely many such congruences.

2. The congruence $\tau(n) \equiv n \sigma(n)(\bmod 5)$ was established by Wilton [110], and is also proved in Hardy's book [50, pp. 166-167]. This congruence was generalized by R. P. Bambah and S. Chowla [15], [33 pp. 676-681], who proved that, if $n$ is not a multiple of 5 , then

$$
\tau(n) \equiv 5 n^{2} \sigma_{7}(n)-4 n \sigma_{9}(n) \quad\left(\bmod 5^{3}\right) .
$$

The asymptotic formula $(2.7)$ can be proved by using the method devised by E. Landau in his book [63, Sect. 183] to determine an asymptotic formula for the number of integers $\leq x$ that can be represented as a sum of two squares. Alternatively, one can appeal to a general Tauberian theorem, such as that proved by H. Delange [37]. However, as first pointed out by G. K. Stanley [102], the claim (2.8) is false. Indeed, by using the ideas of Landau [63, Sects. 176-183], one can establish an asymptotic formula of the shape

$$
\sum_{n \leq x} t_{n}=C \frac{x}{(\log x)^{1 / 4}}\left(1+\sum_{n=1}^{r-1} \frac{c_{n}}{(\log x)^{n}}+O\left(\frac{1}{(\log x)^{r}}\right)\right),
$$


for certain constants $c_{n}, 1 \leq n \leq r-1$. However, generally, these constants are not equal to those which would be obtained by successive integrations by parts in (2.8). Ramanujan made a similar error in his first letter to Hardy [85, p. xxiv], [25, p. 24] when he claimed that the number of integers $\leq x$ that can be represented as a sum of two squares is asymptotic to a constant times a similar integral. See either the sections of Landau's book cited above or Hardy's book [50, pp. 60-63]. In Sections 6, 11, and 17, Ramanujan records similar asymptotic formulas, and, in contrast to the asymptotic formula in this section, calculates the leading coefficients in each case. R. A. Rankin [90] has verified that the leading terms, including the coefficients, are correct in each of the instances cited by Ramanujan.

4. The congruence (4.2) was first proved in print by Wilton [110] and later by Bambah [10].

Rankin [88, p. 5] has pointed out that Ramanujan's conjecture (4.3) is false for $k \geq 4$. Observe that 443 is prime and that its powers are congruent to $\pm 1, \pm 443$ $\left(\bmod 5^{4}\right)$. From Watson's [109] table of values for $\tau(n), \tau(443) \equiv-58\left(\bmod 5^{4}\right)$. Hence, no integers $a$ and $b$ exist for which (4.3) holds with $n=443$ and $k \geq 4$.

However, congruence (4.4) is true and is implied by each of two congruences due to Lahiri [62, eqs. (13.10), (13.11)], namely,

$$
\begin{aligned}
19008 \tau(n) \equiv & -691 \sigma_{13}(n)+27300 \sigma_{11}(n)-691\left\{33(4 n-11) \sigma_{9}(n)\right. \\
& +10(63 n+400) \sigma_{7}(n)-21(860 n-463) \sigma_{5}(n) \\
& \left.+60\left(252 n^{2}-226 n+5\right) \sigma_{3}(n)+(2520 n-431) \sigma(n)\right\} \\
& \left(\bmod 2^{10} \cdot 3^{5} \cdot 5^{3} \cdot 7 \cdot 691\right)
\end{aligned}
$$

and

$$
\begin{aligned}
38016 \tau(n) \equiv & -691 \sigma_{13}(n)+54600 \sigma_{11}(n)-691\left\{33(8 n+19) \sigma_{9}(n)\right. \\
& -30(179 n-200) \sigma_{7}(n)+3\left(2400 n^{2}-5880 n-12299\right) \sigma_{5}(n) \\
& +60\left(120 n^{2}+3039 n-1030\right) \sigma_{3}(n) \\
& \left.-\left(151200 n^{2}-75600 n+6301\right) \sigma(n)\right\}\left(\bmod 2^{11} \cdot 3^{6} \cdot 5^{3} \cdot 7 \cdot 691\right),
\end{aligned}
$$

where, in each case, $n$ is coprime to the modulus. Moreover, (4.4) is equivalent to the congruence

$\tau(n) \equiv 101 n \sigma_{9}(n)+5^{2}\left\{n \sigma_{3}(n)+(n-1) \sigma(n)-\frac{1}{5}\left(n \sigma_{9}(n)-n^{2} \sigma_{7}(n)\right)\right\} \quad\left(\bmod 5^{3}\right)$,

if $(n, 5)=1$, which was stated without proof by Bambah and Chowla [13], [33 pp. 617-619]. The congruence (4.4) is an example of a Borel-type representation; see Serre's exposition in [94].

The equality below (4.4) is a special instance of the relation

$$
\tau\left(p^{n+1}\right)=\tau(p) \tau\left(p^{n}\right)-p^{11} \tau\left(p^{n-1}\right), \quad n>1,
$$

where $p$ is a prime, which along with (7.6), were first proved by L. J. Mordell [65], after Ramanujan had made these conjectures in his paper [80, Sect. 18], [85, p. 153]. 
Proofs of either of the famous equalities (4.5) or (4.6) (or both) have been given by, in chronological order, Ramanujan [81], [85, pp. 210-213], H. B. C. Darling [36], L. J. Mordell [65], H. Rademacher and H. S. Zuckerman [76], [75, pp. 186-202], S. D. Chowla [31], [33 pp. 611-612], D. Kruswijk [61], W. N. Bailey [8], [9], J. M. Dobbie [39], N. J. Fine [41], S. Raghavan [77], H. H. Chan [30], and M. D. Hirschhorn [52], [53]. These proofs are quite varied. Some authors use $q$-series; some, such as Rademacher, Zuckerman, and Raghavan, use the the theory of modular forms; Chan's proof uses a variant of one of Ramanujan's trigonometric series identities in [80].

As indicated by Ramanujan, (4.6) is a companion to (4.5). Bailey, Chan, Darling, Mordell, and Raghavan in the aforementioned papers have also given proofs of (4.6). In contrast to (4.5), equality (4.6) can be found in Ramanujan's notebooks [21, p. 257, Entry 9(i)].

5. Since this section was also extracted by Hardy for [83], we have not added details here.

6. The congruence (6.2) was established by Ramanathan [79], Gupta [49], and Bambah [11].

The comments made in Section 2 about Ramanujan's asymptotic formulas have analogues here. Although the asymptotic formula (6.6a) is correct, Ramanujan's stronger claim (6.7) is false, since the constants obtained by integrating by parts in (6.7) do not generally match those obtained in a proper asymptotic expansion of $\sum_{k=1}^{n} t_{k}$.

7. The content of this section can be found with more detail in Rushforth's paper [92]. (In the second equality of (7.1), Rushforth [92, eq. (7.3)] wrote $-2 R^{2}$ for $2 R^{2}$, and in (7.4) [92, penultimate equality on p. 407] Rushforth wrote $-2 n \sigma_{3}(n)$ for $\left.2 n \sigma_{3}(n).\right)$

8. Ramanujan's proof of (8.1) can be found in his paper [81], [85], while other proofs of (8.1) have been given by Mordell [65], Rademacher and Zuckerman [76], N. J. Fine [41], O. Kolberg [58], Raghavan [77], and Chan [30]. Further identities akin to (8.1) and (8.2) have been established by Rademacher [74], [75, pp. 252-279]. These authors then continue to prove (8.3).

Equality (8.4) is true, and its truth is equivalent to the assertion that $\eta^{3}(z) \eta^{3}(7 z)$ is a Hecke eigenform with complex multiplication in $S_{3}\left(\Gamma_{0}(7), \chi_{-7}\right)$, where $S_{k}\left(\Gamma_{0}(N), \chi\right)$ denotes the complex vector space of cusp forms of weight $k$ with respect to the congruence subgroup $\Gamma_{0}(N)$ with Nebentypus character $\chi$ [91]. (The notation $S_{k}\left(\Gamma_{0}(N)\right)$, with $\chi$ absent, simply means that the character $\chi$ is trivial.) Here the character $\chi_{-7}$ denotes the usual Kronecker character for the field $\mathbb{Q}(\sqrt{-7})$. That this form is an eigenform follows immediately from the fact that this space is one dimensional [35]. To deduce (8.4) in a more elementary fashion, first notice that Jacobi's identity

$$
q \prod_{n=1}^{\infty}\left(1-q^{8 n}\right)^{3}=\eta^{3}(8 z)=\sum_{n=0}^{\infty}(-1)^{n}(2 n+1) q^{(2 n+1)^{2}}
$$

implies that

$$
\eta^{3}(8 z) \eta^{3}(56 z)=\sum_{x, y \geq 0}(-1)^{x+y}(2 x+1)(2 y+1) q^{(2 x+1)^{2}+7(2 y+1)^{2}} .
$$


Proving Ramanujan's claim now follows after a straightforward computation.

The claims regarding the Euler product expansions $\Pi_{1}$ and $\Pi_{2}$ follow easily from the theory of complex multiplication.

In regard to the congruence (8.6), we remark that O. Kolberg [60] proved the beautiful congruence

$$
\tau(n) \equiv n \sigma_{9}(n) \quad(\bmod 49), \quad \text { if }\left(\frac{n}{7}\right)=-1
$$

9. This proof is given in more detail in [83].

10. Equality (10.4) is true, and its truth is equivalent to the assertion that $\eta^{2}(z) \eta^{2}(11 z)$ is an eigenform of the Hecke operators acting on $S_{2}\left(\Gamma_{0}(11)\right)$ [42, p. 432]. That this form is an eigenform follows immediately from the fact that this space is one dimensional.

Some of Ramanujan's congruences for $\tau(n)$ are immediate consequences of its multiplicative properties. For instance, Ramanujan [80], [85, p. 153, eq. (103)] conjectured and Mordell [65] proved that, if $m$ and $n$ are relatively prime integers, then

$$
\tau(m n)=\tau(m) \tau(n)
$$

For example, the congruences (10.8) and (10.9) follow easily since $\tau(19)=10661420$ $\equiv 0(\bmod 11)$ and $\tau(29)=128406630 \equiv 0(\bmod 11)$. Other congruences follow from (C4.1) or from (C4.1) and (C10.1) together.

In 1969, P. Deligne [38] proved Serre's conjecture [95] on the existence of $\ell$-adic Galois representations $\rho_{\ell}$ attached to modular forms on $\Gamma_{0}(N)$. Then, in 1972, Swinnerton-Dyer [103] determined the possible images of $\tilde{\rho}_{\ell}$, the reduction $\bmod \ell$ of $\rho_{\ell}$, and showed that 'small' images imply certain congruences for the coefficients of modular forms.

However in general, the Galois groups which occur are mostly non-abelian (and even non-solvable). In these cases there cannot be a congruence of the form

$$
\tau(p) \equiv c \quad(\bmod \ell)
$$

where the primes $p$ constitute those primes in any given arithmetic progression containing infinitely many primes [103, Lemma 7]. In particular, for $\tau(p)$, such congruences only exist for the primes $\ell \in\{2,3,5,7,23,691\}$. Although this implies that there are no further Ramanujan-type congruences for $\tau(p)$, it turns out that for every positive integer $M$, there is a positive Frobenian set of primes with positive density for which [98]

$$
\tau(p) \equiv 0 \quad(\bmod M) .
$$

This follows from the Chebotarev Density Theorem and the existence of Galois representations. As a consequence, it follows that

$$
\tau(n) \equiv 0 \quad(\bmod M)
$$

for almost every positive integer $n$. 
The existence of these representations and their study has been at the forefront of arithmetic geometry ever since Serre formulated his original conjectures. Every congruence for $\tau(n)$ involving divisor functions, and the congruence

$$
\tau(n) \equiv 0 \quad(\bmod 23)
$$

for $\left(\frac{n}{23}\right)=-1$, follows from this theory. For more details, readers should consult [38], [95], [103], [104], [105], [106].

11. Ramanujan's speculation that $p(n)$ is odd more often than it is even is not substantiated by more extensive calculations. Indeed, it is a long outstanding conjecture that asymptotically $p(n)$ is equally often even and odd. In Sections 1, 5,9 , and 11 , based on a table of values for $p(n), 1 \leq n \leq 200$, computed by P. A. MacMahon, Ramanujan offers conjectures on the distribution of $p(n)$ modulo 5,7 , 11 , and 3, respectively. We examine these conjectures in detail.

If $D(r, M)$ denotes the proportion of integers $n$ for which $p(n) \equiv r(\bmod M)$ (assuming that such densities exist), Ramanujan conjectured (in Sections 11, 11, 1, 5 , and 9 , respectively) that

$$
\begin{aligned}
& D(0,2)<D(1,2), \\
& D(i, 3)=\frac{1}{3}, \quad \text { for } 0 \leq i \leq 2 \text {, } \\
& D(i, 5)= \begin{cases}\frac{1}{3}, & \text { if } i=0, \\
\frac{1}{6}, & \text { if } 1 \leq i \leq 4,\end{cases} \\
& D(i, 7)= \begin{cases}\frac{1}{4}, & \text { if } i=0, \\
\frac{1}{8}, & \text { if } 1 \leq i \leq 6,\end{cases} \\
& D(i, 11)= \begin{cases}\frac{1}{6}, & \text { if } i=0, \\
\frac{1}{12}, & \text { if } 1 \leq i \leq 10 .\end{cases}
\end{aligned}
$$

From elementary considerations, we show that Ramanujan's conjectures for $D(i, M), M=5,7,11$, are unlikely to be true. Remove the values $n=5 k+4,7 k+$ $5,11 k+6$, from consideration when $M=5,7,11$, respectively. Assuming that the remaining values of $p(n)$ are distributed randomly among the $M$ residue classes in each of these three cases, we would expect that

$$
D(i, M)=\left\{\begin{array}{lc}
\frac{2 M-1}{M^{2}}, & \text { if } i=0, \\
\frac{M-1}{M^{2}}, & \text { if } 1 \leq i \leq M
\end{array}\right.
$$

In particular, we expect that $D(0,5)=\frac{9}{25}$ and $D(i, 5)=\frac{4}{25}, 1 \leq i \leq 4$, in contrast to Ramanujan's conjectures. Similar discrepancies exist for $M=7,11$.

Let $\delta(r, M)$ denote the proportion of integers $n \leq 100000$ for which $p(n) \equiv r$ 
$(\bmod M)$. Here are some values of $\delta(r, M)$ for $M \in\{2,3,5,7,11,13\}$.

$\begin{array}{ccccccc}\frac{r}{0} & \frac{\delta(r, 2)}{(0.498} & \frac{\delta(r, 3)}{0.333} & \frac{\delta(r, 5)}{0.362} & \frac{\delta(r, 7)}{0.272} & \frac{\delta(r, 11)}{0.174} & \frac{\delta(r, 13)}{0.080} \\ 1 & 0.502 & 0.332 & 0.158 & 0.121 & 0.083 & 0.078 \\ 2 & * & 0.334 & 0.161 & 0.122 & 0.083 & 0.076 \\ 3 & * & * & 0.160 & 0.122 & 0.082 & 0.077 \\ 4 & * & * & 0.158 & 0.122 & 0.084 & 0.077 \\ 5 & * & * & * & 0.120 & 0.083 & 0.076 \\ 6 & * & * & * & 0.120 & 0.083 & 0.075 \\ 7 & * & * & * & * & 0.081 & 0.077 \\ 8 & * & * & * & * & 0.082 & 0.076 \\ 9 & * & * & * & * & 0.081 & 0.078 \\ 10 & * & * & * & * & 0.082 & 0.075 \\ 11 & * & * & * & * & * & 0.076 \\ 12 & * & * & * & * & * & 0.077\end{array}$

As this data suggests, if the densities $\delta(r, M)$ are well defined, then Ramanujan's conjectures are mostly incorrect. The data suggests that he may be correct when $M=3$, but not for any other values. At present, very little is known about the densities $\delta(r, M)$ apart from lower bounds for $\delta(0, M)$ for those $M$ possessing congruences of the sort discussed in the commentary for Section 1. At present, by the work of S. Ahlgren [1], [2], and J.-L. Nicolas, I. Z. Ruzsa, A. Sárközy, and J.-P. Serre [69], it is known that

$$
\begin{aligned}
& \#\{n \leq X: p(n) \equiv 0(\bmod 2)\} \gg \sqrt{X} \\
& \#\{n \leq X: p(n) \equiv 1(\bmod 2)\} \gg \frac{\sqrt{X}}{\log X} \\
& \#\{n \leq X: p(n) \not \equiv 0(\bmod M)\} \gg \frac{\sqrt{X}}{\log X} .
\end{aligned}
$$

Actually, Serre [69] proved a stronger result, namely,

$$
\frac{\#\{n \leq X: p(a n+b) \equiv 0(\bmod 2)\}}{\sqrt{X}} \rightarrow \infty,
$$

as $X \rightarrow \infty$, for any pair of positive integers $a, b$. On the other hand, the second author [73] has shown that if $M \geq 5$ is prime, then

$$
\#\{n \leq X: p(n) \equiv 0 \quad(\bmod M)\} \gg_{M} X
$$

In a similar direction, M. Newman [68] conjectured that every positive integer $M$ has the property that each residue class $m(\bmod M)$ has infinitely many integers $n$ for which $p(n) \equiv m(\bmod M)$. A. O. L. Atkin [6], O. Kolberg [59], and M. Newman [68] have verified this conjecture for each $M \in\{2,5,7,13\}$. Because of the validity of (14.3) and (14.4), Ramanujan had also proved this conjecture when $M=13$. Motivated by Ramanujan's work, the second author [73] has proved Newman's conjecture for every prime $M<1000$, with the exception of $M=3$. He 
also has found a simple criterion for verifying Newman's conjecture for any prime $M \geq 5$.

The equality (11.4) can be found in a fragment published with Ramanujan's lost notebook [86, p. 354, eq. (1.42)]. A proof may be found in Berndt's paper [23, Entry 21].

The congruence (11.8) has been proved several times in the literature. Most frequently, it is given in the equivalent formulation

$$
\tau(n) \equiv \begin{cases}\sigma(n)(\bmod 3), & \text { if }(3, n)=1, \\ 0(\bmod 3), & \text { if } 3 \mid n\end{cases}
$$

For proofs, see papers by D. P. Banerji [19], Bambah and Chowla [12], [33 pp. 622623], Gupta [48], and Bambah, Chowla, Gupta, and Lahiri [18], [33 pp. 627-630]. Bambah and Chowla [15], [33 pp. 676-681] proved the generalization

$$
\tau(n) \equiv\left(n^{2}+k\right) \sigma_{7}(n) \quad\left(\bmod 3^{4}\right), \quad(3, n)=1
$$

where $k=0$, if $n \equiv 1(\bmod 3)$, and $k=9$, if $n \equiv 2(\bmod 3)$.

The asymptotic formulas in (11.8a) need to be corrected in the same manner that the asymptotic formulas in Sections 2 and 6 needed to be recast.

12. The sums $\sum_{n=1}^{\infty} n^{a} q^{n}$, where $a$ is a positive integer, can be explicitly evaluated in terms of Eulerian polynomials [20, p. 113, Entry 4].

Bambah, Chowla, and Gupta [17], [33 pp. 631-632] and Bambah, Chowla, Gupta, and Lahiri [18], [33 pp. 627-630] proved the congruence

$$
\tau(n) \equiv \sigma(n) \quad(\bmod 8), \quad \text { if } n \text { is odd },
$$

which, in fact, is implied by the first congruence in (12.1).

We have been unable to find the identity (12.2) in the literature prior to the work of Ramanujan. On page 257 in his second notebook [84], Ramanujan actually offers a general formula for

$$
S_{2 r}:=\sum_{n=1}^{\infty} \frac{n^{2 r} q^{n}}{1+q^{n}+q^{2 n}}
$$

which was first proved by Berndt, S. Bhargava, and F. G. Garvan [24], [22, p. 143]. The values of $S_{2 r}, 1 \leq r \leq 4$, are explicitly given by Ramanujan. The formula for $S_{2}$ is given without proof in an equivalent form in a paper by J. M. and P. B. Borwein [28], and this equivalent formula is proved in [29] by the Borweins and Garvan. A particularly simple proof of (12.2), based on an identity of N. J. Fine, has been given by S. H. Son [101, Lemma 2.6].

A proof of the first congruence in (12.3) was given by Bambah and Chowla [14], [33 pp. 633-634]. The second congruence in (12.3) was established in another paper by the same authors [15], [33 pp. 676-681].

Bambah and Chowla [14], [33 pp. 633-634] proved the second congruence in (12.4).

The first proof in print of (12.7) was evidently given by J. R. Wilton [110]. Later proofs were found by G. N. Watson [107] and D. H. Lehmer [64]. 
As with corresponding results in Sections 2, 6, and 11, the asymptotic formula (12.7a) needs to be corrected.

Bambah and Chowla [16], [33 pp. 644-651] gave the first published proof of (12.9).

The congruence below (12.9) is false, in general. For example, it is false for $n=1,3,4,5$.

13. In Sections $2,6,10,11$, and 13 Ramanujan considers the $t$-regular partition functions $\lambda(n)$ whose generating function are given by

$$
\sum_{n=0}^{\infty} \lambda(n) q^{n}=\sum_{n=0}^{\infty} b_{t}(n) q^{n}:=\prod_{n=1}^{\infty} \frac{\left(1-q^{t n}\right)}{\left(1-q^{n}\right)} .
$$

The dependence of $\lambda$ on $t$ is always clear from the context. For instance, in Section 2, he considers the case where $t=25$. In this case he shows that $\lambda(n)$ is almost always a multiple of 5. A recent paper by B. Gordon and the second author [46] makes considerable progress in describing this phenomenon for all $t$. Let $p_{1}^{a_{1}} p_{2}^{a_{2}} \cdots p_{m}^{a_{m}}$ be the prime factorization of $t$. By [46, Thm. 1], if $p_{i}$ is a prime for which $p_{i}^{a_{i}} \geq \sqrt{t}$, then, for every positive integer $k$, almost every integer $n$ has the property that $b_{t}(n)$ is a multiple of $p_{i}^{k}$. This theorem immediately implies all of Ramanujan's claims of this sort for the functions $\lambda(n)$.

Equality (13.6) has been proved by H. S. Zuckerman [112] and W. H. Simons [100].

14. The claims (14.1)-(14-6) are among the most fascinating results in the unpublished manuscript. For example, these results indicate that

$$
\sum_{n=0}^{\infty} p(13 n+6) q^{24 n+11} \equiv 11 \eta^{11}(24 z) \quad(\bmod 13) .
$$

M. Newman [67] has proved some of these claims. However, the second author [73] has shown that this phenomenon also holds with respect to other moduli. In particular, if $m \geq 5$ is prime and $k$ is a positive integer, then

$$
\sum_{n=0}^{\infty} p\left(\frac{m^{k} n+1}{24}\right) q^{n}
$$

is the reduction modulo $m$ of a holomorphic cusp form of weight $\frac{m^{2}-m-1}{2}$. This implies that results like (14.1)-(14.6) exist for every prime $m \geq 5$, not just $m=13$. Moreover, using the theory of Hecke operators of half-integral weight, the Shimura correspondence, and the theory of Galois representations, the second author [73] has proved that for every prime $m \geq 5$ that there are integers $0 \leq b<a$ for which

$$
p(a n+b) \equiv 0 \quad(\bmod m)
$$

for every non-negative integer $n$.

15. In Section 15 Ramanujan gives a brief description of the method he employs to obtain generating functions of the type

$$
\sum p\left(\varpi n+b_{\varpi}\right) q^{n} \quad(\bmod \varpi)
$$


where $\varpi>3$ is prime. Let $B_{k}$ denote the $k$ th Bernoulli number in contemporary notation. Note that Ramanujan's convention for Bernoulli numbers is different from the contemporary one in which the Bernoulli numbers $B_{k}$ are defined by

$$
\frac{x}{e^{x}-1}=\sum_{k=0}^{\infty} \frac{B_{k}}{k !} x^{k}, \quad|x|<2 \pi .
$$

For every positive even integer $k$, define positive coprime integers $v_{k}$ and $\delta_{k}$ by

$$
\frac{v_{k}}{\delta_{k}}:=\left|B_{k}\right|
$$

If $k \geq 4$ is an even integer, let

$$
E_{2 k}(z):=1-\frac{4 k}{B_{2 k}} \sum_{n=1}^{\infty} \sigma_{2 k-1}(n) q^{n}
$$

denote the normalized Eisenstein series of weight $2 k$ with respect to the full modular group $\Gamma_{0}(1)$, where $B_{k}$ is defined by (C15.1).

Now assume that $\varpi>3$ is prime. It is easy to check that the left hand side of (15.1) is $v_{\varpi-1} \cdot E_{\varpi-1}(z)$ and that the left hand side of (15.2) represents $v_{\varpi+1}$. $E_{\varpi+1}(z)$. That both of these $q$-series have integer coefficients is obvious, and that they have the desired representation as sums of $Q^{\ell} R^{m}$ is well known [89, p. 199, Thm. 6.1.3]. In fact, every holomorphic modular form with respect to the full modular group has such a representation.

As Ramanujan claims, (15.3) and (15.31) are easy deductions from Fermat's Little Theorem and the von Staudt and Claussen theorem. However, the claim that $k=0$ in (15.31) is not entirely clear. In fact, this is one of the questions in the manuscript that Ramanujan admits still requires proof.

Proposition 1. Ramanujan's claim that $k \equiv 0(\bmod \varpi)$ in $(15.31)$ is true.

Proof. A simple calculation verifies Ramanujan's assertion that the truth of (15.4) implies that $k$ is indeed 0 , or more precisely $0(\bmod \varpi)$. In particular, it suffices to prove that

$$
12 v_{\varpi+1}+(-1)^{\frac{\varpi+1}{2}} \delta_{\varpi+1} \equiv 0 \quad(\bmod \varpi)
$$

Using the well known Voronoi congruences [55, p. 237, Prop. 15.2.3], we find, for every integer $a$ coprime to $\varpi$, that

$$
\left(a^{2}-1\right) v_{\varpi+1} \equiv a \cdot(-1)^{\frac{\varpi-1}{2}} \delta_{\varpi+1} \sum_{j=1}^{\varpi-1} j\left[\frac{j a}{\varpi}\right] \quad(\bmod \varpi),
$$

since the sign of $B_{2 k}$ is $(-1)^{k+1}$ for every positive integer $k$. Therefore we find that

$$
\left(\frac{a^{2}-1}{a}\right) v_{\varpi+1}+(-1)^{\frac{\varpi+1}{2}} \delta_{\varpi+1} \sum_{j=1}^{\varpi-1} j\left[\frac{j a}{\varpi}\right] \equiv 0 \quad(\bmod \varpi)
$$


In view of (C15.2) and (C15.3), it suffices to prove that, for each integer $a$ coprime to $\varpi$

$$
\sum_{j=1}^{\varpi-1} j\left[\frac{j a}{\varpi}\right] \equiv \frac{a^{2}-1}{12 a} \quad(\bmod \varpi) .
$$

We now prove (C15.4) by examining Dedekind sums. If $k$ is a positive integer, and $h$ is coprime to $k$, then the Dedekind sum $s(h, k)$ is defined by

$$
s(h, k):=\sum_{j=1}^{k-1} \frac{j}{k}\left(\frac{h j}{k}-\left[\frac{h j}{k}\right]-\frac{1}{2}\right) .
$$

It is easy to verify that

$$
12 a \varpi s(a, \varpi)=\frac{12 a^{2}}{\varpi} \cdot \frac{\varpi(\varpi-1)(2 \varpi-1)}{6}-12 a \sum_{j=1}^{\varpi-1} j\left[\frac{j a}{\varpi}\right]-6 a \cdot \frac{\varpi(\varpi-1)}{2} .
$$

However, by $[4$, p. 64 , Th. 3.8], it is known that

$$
12 a \varpi s(a, \varpi) \equiv a^{2}+1 \quad(\bmod \varpi),
$$

and so we find that

$$
a^{2}+1 \equiv 2 a^{2}-12 a \sum_{j=1}^{\varpi-1} j\left[\frac{j a}{\varpi}\right] \quad(\bmod \varpi) .
$$

This is (C15.4), and this completes the proof of Ramanujan's claim.

A proof of the previous proposition has also been given by Swinnerton-Dyer [103, Theorem 2(i)].

In Section 15, Ramanujan asserted that, in particular cases, he was able to show that (see (15.6))

\section{Proposition 2.}

$$
\left(Q^{3}-R^{2}\right)^{\left(\varpi^{2}-1\right) / 24} \equiv q \frac{d J}{d q}+F \quad(\bmod \varpi),
$$

where $F$ is a cusp form of weight $\varpi-1$ on $S L_{2}(\mathbb{Z})$.

As a corollary, he deduced that (see (15.9))

Proposition 3. The function

$$
\left(q^{\varpi} ; q^{\varpi}\right)_{\infty} \sum_{n=0}^{\infty} p\left(\varpi n+\frac{1-\varpi^{2}}{24}\right) q^{n} \equiv F \quad(\bmod \varpi),
$$

where $F$ is a cusp form of weight $\varpi-1$ on $S L_{2}(\mathbb{Z})$.

Since the space of cusp forms on $S L_{2}(\mathbb{Z})$ of weight $\varpi-1$ for $\varpi \leq 11$ is trivial, Proposition 3 immediately implies the congruences

$p(5 n+4) \equiv 0(\bmod 5), \quad p(7 n+5) \equiv 0(\bmod 7) \quad$ and $\quad p(11 n+6) \equiv 0(\bmod 11)$. 
A proof of Proposition 2 for general $\varpi$ has been given by Serre using [96, Lemma 2]. Proposition 3 was recently rediscovered and proved by K. S. Chua [34] without using Proposition 2.

The remainder of Section 15 is straightforward and follows from Ramanujan's collection of formulas involving the operator $q \frac{d}{d q}$.

16. The equalities (16.3) and (16.7) are proved in Rushforth's paper [92]. As with the key results in Section 14, the claims (16.1)-(16.3) and (16.5)-(16.9) follow from the work of Ono [73].

In (16.4), Ramanujan claims that the Dirichlet series

$$
\begin{array}{ll}
\sum_{n=1}^{\infty} \frac{\tau_{2}(n)}{n^{s}}, & \sum_{n=1}^{\infty} \frac{\tau_{3}(n)}{n^{s}}, \\
\sum_{n=1}^{\infty} \frac{\tau_{4}(n)}{n^{s}}, & \sum_{n=1}^{\infty} \frac{\tau_{5}(n)}{n^{s}}, \quad \sum_{n=1}^{\infty} \frac{\tau_{7}(n)}{n^{s}}
\end{array}
$$

have Euler products. This is easily verified since all corresponding modular forms are eigenforms of Hecke operators, for they each lie in a one dimensional space of cusp forms [35].

At the end of Section 16, Ramanujan claims that the two Dirichlet series

$$
\sum_{n=1}^{\infty} \frac{\Omega_{2}(n)}{n^{s}} \text { and } \sum_{n=1}^{\infty} \frac{\Omega_{3}(n)}{n^{s}}
$$

are both differences of two series with Euler products. In terms of classical modular forms, $\sum_{n=1}^{\infty} \Omega_{2}(n) q^{n} \in S_{28}\left(\Gamma_{0}(1)\right)$ and $\sum_{n=1}^{\infty} \Omega_{3}(n) q^{n} \in S_{30}\left(\Gamma_{0}(1)\right)$. Both of these two spaces are two dimensional [35], and one can easily check that

$$
\begin{aligned}
& S_{28}\left(\Gamma_{0}(1)\right)=\mathbb{C} Q \Delta^{2} \oplus \mathbb{C} Q^{4} \Delta, \\
& S_{30}\left(\Gamma_{0}(1)\right)=\mathbb{C} R \Delta^{2} \oplus \mathbb{C} R^{3} \Delta,
\end{aligned}
$$

where $\Delta:=\Delta(q)=q(q ; q)_{\infty}^{24}$. It is easy to show that the space $S_{28}\left(\Gamma_{0}(1)\right)$ is spanned by the eigenforms

$$
\begin{aligned}
& f_{1}:=(-5076+108 \sqrt{18209}) Q \Delta^{2}+Q^{4} \Delta, \\
& f_{2}:=(-5076-108 \sqrt{18209}) Q \Delta^{2}+Q^{4} \Delta .
\end{aligned}
$$

(For calculations of this sort, see N. Koblitz's text [57, p. 173, Prop. 51].) Since $\sum_{n=1}^{\infty} a_{1}(n) q^{n}$ and $\sum_{n=1}^{\infty} a_{2}(n) q^{n}$ are eigenforms, the two Dirichlet series

$$
\sum_{n=1}^{\infty} \frac{a_{1}(n)}{n^{s}} \text { and } \sum_{n=1}^{\infty} \frac{a_{2}(n)}{n^{s}}
$$

have Euler products as in (16.4) with weight 28. The "difference" to which Ramanujan alludes is the identity

$$
Q \Delta^{2}=\sum_{n=1}^{\infty} \Omega_{2}(n) q^{n}=\frac{1}{216 \sqrt{18209}}\left(f_{1}-f_{2}\right) .
$$


Similarly, one can easily verify that the space $S_{30}\left(\Gamma_{0}(1)\right)$ is spanned by the eigenforms

$$
\begin{aligned}
& g_{1}:=(5856+96 \sqrt{51349}) R \Delta^{2}+R^{3} \Delta, \\
& g_{2}:=(5856-96 \sqrt{51349}) R \Delta^{2}+R^{3} \Delta .
\end{aligned}
$$

Since $\sum_{n=1}^{\infty} b_{1}(n) q^{n}$ and $\sum_{n=1}^{\infty} b_{2}(n) q^{n}$ are eigenforms, it easily follows that the two Dirichlet series

$$
\sum_{n=1}^{\infty} \frac{b_{1}(n)}{n^{s}} \text { and } \sum_{n=1}^{\infty} \frac{b_{2}(n)}{n^{s}}
$$

have Euler products as in (16.4) with weight 30. The difference to which Ramanujan alludes is the identity

$$
R \Delta^{2}=\sum_{n=1}^{\infty} \Omega_{3}(n) q^{n}=\frac{1}{192 \sqrt{51349}}\left(g_{1}-g_{2}\right) .
$$

17. The claim (17.2) is equivalent to the assertion that $\eta(z) \eta(23 z)$ is an eigenform with complex multiplication in the space $S_{1}\left(\Gamma_{0}(23), \chi_{-23}\right)$ [42, p. 472], [47], [91], [35]. Here $\chi_{-23}$ is the usual Kronecker character for the quadratic field $\mathbb{Q}(\sqrt{-23})$. Although the claims regarding the Euler products $\Pi_{1}, \Pi_{2}$ and $\Pi_{3}$ follow easily from the theory of complex multiplication, one can more easily obtain them from Euler's pentagonal number theorem,

$$
(q ; q)_{\infty}=\sum_{n=-\infty}^{\infty}(-1)^{n} q^{n(3 n+1) / 2} .
$$

Here one would use an argument similar to that briefly outlined in the commentary for Section 8. In analogy with our comments in Sections 2 and 6, the claim (17.8) is false, but the leading term in the asymptotic expansion is indeed $C x / \sqrt{\log x}$.

18. The proof of (18.1) is quite difficult, but it is given in Rushforth's paper [92]. Ramanujan has omitted many details in his assertion (18.2). For the remainder of the proof of Ramanujan's congruence modulo $11^{2}$ to be completed, it is necessary to explicitly determine the constants $a_{4}, b_{4}$, and $c_{3}$ in (18.2). Rushforth does not prove (18.2) but proceeds by a different route to (18.7). The third congruence in (18.3) is proved in Rushforth's paper [92]. The equation to which Ramanujan refers before (18.7) is not given in the manuscript, but would arise from (18.2) by using (18.3)-(18.6).

19. This manuscript contains many results on the divisibility of $\tau(n)$. In several sections Ramanujan concludes that $\tau(n)$ is a multiple of a given integer $M$ for almost all $n$. In other words, for such $M$,

$$
\lim _{X \rightarrow \infty} \frac{\#\{1 \leq n \leq X: \tau(n) \equiv 0(\bmod M)\}}{X}=1
$$

Specifically, Ramanujan finds in (19.9) that $\tau(n)$ is a multiple of $2^{5} \cdot 3^{3} \cdot 5^{2} \cdot 7^{2} \cdot 23$. 691 for almost all $n$. Various authors have proved versions of (19.9) with varying 
exponents on the six primes. It was first proved by Chowla [32], [33 pp. 639-643] that, in fact, the conclusion still holds if the powers of $2,3,5,7,23$, and 691 are replaced by any set of six positive integral powers.

Ramanujan obtains his results by employing the congruences for $\tau(n)$ with modulus $M \in\left\{2^{5}, 3^{3}, 5^{2}, 7^{2}, 23,691\right\}$. In each case, he finds that a positive density of primes $p$ has the property that $\tau(p) \equiv 0(\bmod M)$. A Tauberian argument based on the multiplicativity of $\tau(n)$ then leads to his conclusion [98, Sect. 2].

Results of this type depend upon the divisibility of divisor functions. Improving on Watson's theorem [107], Rankin [87] found an asymptotic formula for the number of positive integers $\leq x$ for which $\sigma_{\nu}(n)$ is not divisible by the prime number $k$. These results were generalized by E. J. Scourfield [93].

J.-P. Serre [97], [98], [99] has obtained a substantial generalization of Ramanujan's claims for all modular forms of integral weight with respect to congruence subgroups of the full modular group. In particular, if $\sum_{n=1}^{\infty} a(n) q^{n}\left(q:=e^{2 \pi i z}\right)$ is the Fourier expansion of a modular form of integral weight with integral coefficients, then for every positive integer $M, a(n) \equiv 0(\bmod M)$ for almost all $n$. M. R. Murty and V. K. Murty [66] have obtained an interesting improvement on the original formulation of Serre's result.

Serre's theorem is based on the existence of $\ell$-adic Galois representations associated to modular forms (see the comments on Section 10). In addition to providing an arithmetic and group theoretic description of congruences for Fourier coefficients $a(n)$ of the types found by Ramanujan for $\tau(n)$, their mere existence implies, by the Chebotarev Density Theorem, that a positive proportion of primes $p$ have the property that $a(p) \equiv 0(\bmod M)$.

Bambah and Chowla [13], [33 pp. 617-619] state without proofs several congruences for $\tau(n)$. Lahiri [62] gives an enormous number of congruences involving $\tau(n)$. Van der Blij's beautiful paper [27], giving congruences and other properties of $\tau(n)$, is particularly recommended. Except for those employing the theory of $\ell$-adic Galois representations, almost all the authors giving proofs of congruences for $\tau(n)$ whom we have cited use ideas similar to those employed by Ramanujan in this manuscript.

20.-23. These sections contain Ramanujan's proof of his congruence for $p(n)$ modulo any positive integral power of 5 , with (22.5)-(22.8) being the principal congruences. Observe that (22.7) and (22.8) include (C1.1). As mentioned earlier, the ideas here were expanded into a more detailed proof given in 1938 by Watson [108], who does not mention Part II of Ramanujan's unpublished manuscript in his paper, although according to Rushforth [92], Watson received a copy from Hardy in 1928.

The details in Section 20 are reasonably ample, but beginning with Section 21, the details are sparse. In particular, (21.1) is difficult to prove. The proof given by Watson may follow along somewhat different lines than those indicated by Ramanujan. Readers can likely follow the details for the remainder of Section 21. We have added some details for (21.6), which is not used in Watson's work. The heart of Ramanujan's proof lies in (22.1)-(22.6), for which Ramanujan provides no details. These are developed in Watson's paper [108].

24. Clearly, Ramanujan intended to follow the same lines of attack for powers of 7 as he did for powers of 5 in Sections 20-23. If he had completed his argument, he would have undoubtedly seen that his original conjecture modulo powers of 7 needed 
to be corrected. Most likely, his declining health prevented him from working out the remaining details, which were completed by Watson [108].

To verify the equations (24.4)-(24.6), it suffices to notice that all three equations are essentially claims about the presentation of modular functions with respect to $\Gamma_{0}(7)$. In each case, one may multiply both sides of the claimed identity by $\left(q^{7} ; q^{7}\right)^{8}$. After doing so, one needs to compare, up to a shifted power of $q$, the Fourier expansions of two cusp forms of weight 4 . One can then easily deduce these claims from the results in [35].

Ramanujan's proof of (24.6a) is quite elementary, but the algebraic manipulations are a bit tedious. A similar, and perhaps more efficient, proof of (24.6a) has been given by O. Kolberg [58].

At the end of Part II are two detatched fragments. They actually appear at the end of Section 21 in Watson's copy of the manuscript, but it seems to us that they are better placed at the end of the section pertaining to the moduli 7 and 49 .

We are grateful to Paul Bateman, Heng Huat Chan, Michael Hirschhorn, Robert A. Rankin, and Jean-Pierre Serre for helpful comments.

\section{REFERENCES}

1. S. Ahlgren, Distribution of parity of the partition function in arithmetic progressions, Indag. Math. 10 (1999), 173-181.

2. S. Ahlgren, Non-vanishing of the partition function modulo odd primes $\ell$, Mathematika 46 (1999), 185-192.

3. G. E. Andrews and F. G. Garvan, Dyson's crank of a partition, Bull. Amer. Math. Soc. 18 (1988), 167-171.

4. T. Apostol, Modular Functions and Dirichlet Series in Number Theory, Springer-Verlag, New York, 1990.

5. A. O. L. Atkin, Proof of a conjecture of Ramanujan, Glasgow Math. J. 8 (1967), 14-32.

6. A. O. L. Atkin, Multiplicative congruence properties and density problems for $p(n)$, Proc. London Math. Soc. 18 (1968), 563-576.

7. A. O. L. Atkin and J. N. O'Brien, Some properties of $p(n)$ and $c(n)$ modulo powers of 13, Trans. Amer. Math. Soc. 126 (1967), 442-459.

8. W. N. Bailey, A note on two of Ramanujan's formulae, Quart. J. Math. Oxford 3 (1952), 29-31.

9. W. N. Bailey, A further note on two of Ramanujan's formulae, Quart. J. Math. Oxford 3 (1952), 158-160.

10. R. P. Bambah, Two congruence properties of Ramanujan's function $\tau(n)$, J. London Math. Soc. 21 (1946), 91-93.

11. R. P. Bambah, Ramanujan's function $\tau(n)-$ a congruence property, Bull. Amer. Math. Soc. 53 (1947), 764-765.

12. R. P. Bambah and S. Chowla, A congruence property of Ramanujan's function $\tau(n)$, Proc. Nat. Inst. Sci. India 12 (1946), 431-432.

13. R. P. Bambah and S. Chowla, Some new congruence properties of Ramanujan's function $\tau(n)$, Math. Student 14 (1946), 24-26.

14. R. P. Bambah and S. Chowla, A new congruence property of Ramanujan's function $\tau(n)$, Bull. Amer. Math. Soc. 53 (1947), 768-769.

15. R. P. Bambah and S. Chowla, Congruence properties of Ramanujan's function $\tau(n)$, Bull. Amer. Math. Soc. 53 (1947), 950-955.

16. R. P. Bambah and S. Chowla, The residue of Ramanujan's function $\tau(n)$ to the modulus $2^{8}$, J. London Math. Soc. 22 (1947), 140-147.

17. R. P. Bambah, S. Chowla, and H. Gupta, A congruence property of Ramanujan's function $\tau(n)$, Bull. Amer. Math. Soc. 53 (1947), 766-767.

18. R. P. Bambah, S. Chowla, H. Gupta, and D. B. Lahiri, Congruence properties of Ramanujan's function $\tau(n)$, Quart. J. Math. 18 (1947), 143-146. 
19. D. P. Banerji, Congruence properties of Ramanujan's function $\tau(n)$, J. London Math. Soc. 17 (1942), 144-145.

20. B. C. Berndt, Ramanujan's Notebooks, Part I, Springer-Verlag, New York, 1985.

21. B. C. Berndt, Ramanujan's Notebooks, Part III, Springer-Verlag, New York, 1991.

22. B. C. Berndt, Ramanujan's Notebooks, Part V, Springer-Verlag, New York, 1998.

23. B. C. Berndt, Fragments by Ramanujan on Lambert series, Number Theory and Its Applications (K. Györy and S. Kanemitsu, eds.), Kluwer, Dordrecht, 1999, pp. 35-49..

24. B. C. Berndt, S. Bhargava, and F. G. Garvan, Ramanujan's theories of elliptic functions to alternative bases, Trans. Amer. Math. Soc. 347 (1995), 4163-4244.

25. B. C. Berndt and R. A. Rankin, Ramanujan: Letters and Commentary, American Mathematical Society, Providence, 1995; London Mathematical Society, London, 1995.

26. B. J. Birch, A look back at Ramanujan's notebooks, Proc. Cambridge Philos. Soc. 78 (1975), 73-79.

27. F. van der Blij, The function $\tau(n)$ of $S$. Ramanujan (an expository lecture), Math. Student 18 (1950), 83-99.

28. J. M. and P. B. Borwein, A cubic counterpart of Jacobi's identity and the AGM, Trans. Amer. Math. Soc. 323 (1991), 691-701.

29. J. M. Borwein, P. B. Borwein, and F. G. Garvan, Some cubic modular identities of Ramanujan, Trans. Amer. Math. Soc. 343 (1994), 35-47.

30. H. H. Chan, New proofs of Ramanujan's partition identities for moduli 5 and 7, J. Number Theory 53 (1995), 144-159.

31. S. Chowla, Outline of a new method for proving results of elliptic function theory (such as identities of the Ramanujan-Rademacher-Zuckerman type), Proc. Lahore Philos. Soc. 7 (1945), 45-55.

32. S. Chowla, On a theorem of Walfisz, J. London Math. Soc. 22 (1947), 136-140.

33. S. Chowla, The Collected Papers of Sarvadaman Chowla, Volume II (J. G. Huard and K. S. Williams, eds.), Les Publications CRM, Montreal, 1999.

34. K. S. Chua, Explicit congruences of the partition function modulo every prime; preprint.

35. H. Cohen and J. Oesterlé, Dimensions des espaces de formes modulaires, Modular Functions of One Variable VI, Lecture Notes in Math., No. 627, Springer-Verlag, Berlin, 1977, pp. 69-78.

36. H. B. C. Darling, Proofs of certain identities and congruences enunciated by S. Ramanujan, Proc. London Math. Soc. (2) 19 (1921), 350-372.

37. H. Delange, Généralisation du théorème de Ikehara, Ann. Sci. Ecole Norm. Sup. (3) 71 (1954), 213-242.

38. P. Deligne, Formes modulaires et représentations $\ell$-adiques, Séminaire Bourbaki (1968/1969), Exp. No. 355, Lecture Notes in Math., No. 179, Springer-Verlag, Berlin, 1971, pp. 139-172.

39. J. M. Dobbie, A simple proof of some partition formulae of Ramanujan's, Quart. J. Math. Oxford 6 (1955), 193-196.

40. F. J. Dyson, Some guesses in the theory of partitions, Eureka (Cambridge) 8 (1944), $10-15$.

41. N. J. Fine, On a system of modular functions connected with the Ramanujan identities, Tôhoku J. Math. 8 (1956), 149-164.

42. R. Fricke, Lehrbuch der Algebra, Bd. 3, F. Vieweg, Braunschweig, 1928.

43. F. G. Garvan, A simple proof of Watson's partition congruences for powers of 7, J. Austral. Math. Soc. 36 (1984), 316-334.

44. F. G. Garvan, New combinatorial interpretations of Ramanujan's partition congruences $\bmod 5$, 7, and 11, Trans. Amer. Math. Soc. 305 (1988), 47-77.

45. F. G. Garvan, D. Kim, and D. Stanton, Cranks and t-cores, Invent. Math. 101 (1990), $1-17$.

46. B. Gordon and K. Ono, Divisibility of certain partition functions by powers of primes, The Ramanujan J. 1 (1997), 25-34.

47. B. Gordon and D. Sinor, Multiplicative properties of $\eta$-products, Lecture Notes in Math., No. 1395, Springer-Verlag, New York, 1988, pp. 173-200.

48. H. Gupta, A congruence relation between $\tau(n)$ and $\sigma(n)$, J. Indian Math. Soc. 9 (1945), 59-60.

49. H. Gupta, A congruence property of $\tau(n)$, Proc. Indian Acad. Sci. A 24 (1946), 441-442.

50. G. H. Hardy, Ramanujan, Chelsea, New York, 1978. 
51. G. H. Hardy and E. M. Wright, An Introduction to the Theory of Numbers, 4 th ed., Clarendon Press, Oxford, 1960.

52. M. D. Hirschhorn, An identity of Ramanujan, and applications, $q$-series from a Contemporary Perspective (M. E. H. Ismail and D. Stanton, eds.), American Mathematical Society, Providence, RI, 2000, pp. 229-234.

53. M. D. Hirschhorn, Another short proof of Ramanujan's mod 5 partition congruence, and more, Amer. Math. Monthly 106 (1999), 580-583.

54. M. D. Hirschhorn and D. C. Hunt, A simple proof of the Ramanujan conjecture for powers of 5, J. Reine Angew. Math. 336 (1981), 1-17.

55. K. Ireland and M. Rosen, A Classical Introduction to Modern Number Theory, SpringerVerlag, New York, 1982.

56. M. I. Knopp, Modular Functions in Analytic Number Theory, Chelsea, New York, 1993.

57. N. Koblitz, Introduction to Elliptic Curves and Modular Forms, Springer-Verlag, New York, 1984.

58. O. Kolberg, Some identities involving the partition function, Math. Scand. 5 (1957), 7792.

59. O. Kolberg, Note on the parity of the partition function, Math. Scand. 7 (1959), 377-378.

60. O. Kolberg, Note on Ramanujan's function $\tau(n)$, Math. Scand. 10 (1962), 171-172.

61. D. Kruyswijk, On some well-known properties of the partition function $p(n)$ and Euler's infinite product, Nieuw Arch. Wisk. 23 (1950), 97-107.

62. D. B. Lahiri, On Ramanujan's function $\tau(n)$ and the divisor function $\sigma_{k}(n)$, II, Bull. Calcutta Math. Soc. 39 (1947), 33-52.

63. E. Landau, Handbuch der Lehre von der Verteilung der Primzahlen, Chelsea, New York, 1953.

64. D. H. Lehmer, Some functions of Ramanujan, Math. Student 27 (1959), 105-116.

65. L. J. Mordell, Note on certain modular relations considered by Messrs Ramanujan, Darling and Rogers, Proc. London Math. Soc. 20 (1922), 408-416.

66. M. R. Murty and V. K. Murty, Prime divisors of Fourier coefficients of modular forms, Duke Math. J. 51 (1984), 57-76.

67. M. Newman, Congruences for the coefficients of modular forms and some new congruences for the partition function, Canad. J. Math. 9 (1957), 549-552.

68. M. Newman, Periodicity modulo $m$ and divisibility properties of the partition function, Trans. Amer. Math. Soc. 97 (1960), 225-236.

69. J.-L. Nicolas, I. Z. Ruzsa, and A. Sárközy, On the parity of additive representation functions, with an appendix by J.-P. Serre, J. Number Theory 73 (1998), 292-317.

70. K. Ono, On the parity of the partition function, J. Reine Angew. Math. 472 (1996), 1-15.

71. K. Ono, The residue of $p(n)$ modulo small primes, The Ramanujan J. 2 (1998), 47-54.

72. K. Ono, The partition function in arithmetic progressions, Math. Ann. 312 (1998), 251260 .

73. K. Ono, Distribution of the partition function modulo m, Ann. of Math. 151 (2000), 293-307.

74. H. Rademacher, The Ramanujan identities under modular substitutions, Trans. Amer. Math. Soc. 51 (1942), 609-636.

75. H. Rademacher, Collected Papers of Hans Rademacher, Vol. II, MIT Press, Cambridge, MA, 1974.

76. H. Rademacher and H. S. Zuckerman, A new proof of two of Ramanujan's identities, Ann. of Math. 40 (1939), 473-489.

77. S. Raghavan, On certain identities due to Ramanujan, Quart. J. Math. Oxford 37 (1986), 221-229.

78. K. G. Ramanathan, Congruence properties of Ramanujan's function $\tau(n)$, Proc. Indian Acad. Sci. A 19 (1944), 146-148.

79. K. G. Ramanathan, Congruence properties of Ramanujan's function $\tau(n)$, II, J. Indian Math. Soc. 9 (1945), 55-59.

80. S. Ramanujan, On certain arithmetical functions, Trans. Cambridge Philos. Soc. 22 (1916), 159-184.

81. S. Ramanujan, Some properties of $p(n)$, the number of partitions of $n$, Proc. Cambridge Philos. Soc. 19 (1919), 207-210. 
82. S. Ramanujan, Congruence properties of partitions, Proc. London Math. Soc. 18 (1920), xix.

83. S. Ramanujan, Congruence properties of partitions, Math. Z. 9 (1921), 147-153.

84. S. Ramanujan, Notebooks (2 volumes), Tata Institute of Fundamental Research, Bombay, 1957.

85. S. Ramanujan, Collected Papers, Chelsea, New York, 1962.

86. S. Ramanujan, The Lost Notebook and Other Unpublished Papers, Narosa, New Delhi, 1988.

87. R. A. Rankin, The divisibility of divisor functions, Glasgow Math. J. 5 (1961), 35-40.

88. R. A. Rankin, Ramanujan's unpublished work on congruences, Modular Functions of One Variable V, Lecture Notes in Math., No. 601, Springer-Verlag, Berlin, 1977, pp. 3-15.

89. R. A. Rankin, Modular Forms and Functions, Cambridge University Press, Cambridge, 1977.

90. R. A. Rankin, Ramanujan's manuscripts and notebooks, Bull. London Math. Soc. 14 (1982), 81-97.

91. K. Ribet, Galois representations attached to eigenforms with Nebentypus, Modular Functions of One Variable V, Lecture Notes in Math., No. 601, Springer-Verlag, Berlin, 1977, pp. $17-51$.

92. J. M. Rushforth, Congruence properties of the partition function and associated functions, Proc. Cambridge Philos. Soc. 48 (1952), 402-413.

93. E. J. Scourfield, On the divisibility of $\sigma_{\nu}(n)$, Acta Arith. 10 (1964), 245-285.

94. J.-P. Serre, Une interprétation des congruences relatives à la fonction $\tau$ de Ramanujan, Séminaire Delange-Pisot-Poitou: 1967/68, Théorie des Nombres, Fasc. 1, Exp. 14, Secrétariat Mathématique, Paris, 1969, 17 pp..

95. J.-P. Serre, Congruences et formes modulaires [d'après H. P. F. Swinnerton-Dyer], Séminaire Bourbaki, 24e année (1971/1972), Exp. No. 416, Lecture Notes in Math., No. 317, SpringerVerlag, Berlin, 1973, pp. 319-338.

96. J.-P. Serre, Formes modulaires et fonctions zêta p-adiques, Modular Functions of One Variable III, Lecture Notes in Math. No. 350, Springer-Verlag, Berlin, 1973, pp. 191-268.

97. J.-P. Serre, Divisibilité des coefficients des formes modulaires de poids entier, C. R. Acad. Sci. (Paris), Sér A 279 (1974), 670-682.

98. J.-P. Serre, Divisibilité de certaines fonctions arithmétiques, L'Enseign. Math. 22 (1976), $227-260$.

99. J.-P. Serre, Quelques applications du théoréme de densité de Chebotarev, Publ. Math. I. H. E. S. 54 (1981), 123-201.

100. W. H. Simons, Congruences involving the partition function $p(n)$, Bull. Amer. Math. Soc. 50 (1944), 883-892.

101. S. H. Son, Some integrals of theta functions in Ramanujan's lost notebook, Number Theory, Fifth Conference of the Canadian Number Theory Association (R. Gupta and K. S. Williams, eds.), American Mathematical Society, Providence, RI, 1998, pp. 329-339.

102. G. K. Stanley, Two assertions made by Ramanujan, J. London Math. Soc. 3 (1928), 232-237.

103. H. P. F. Swinnerton-Dyer, On $\ell$-adic representations and congruences for coefficients of modular forms, Modular Functions of One Variable III, Lecture Notes in Math., No. 350, Springer-Verlag, Berlin, 1973, pp. 1-55.

104. H. P. F. Swinnerton-Dyer, Correction to: "On $\ell$-adic representations and congruences for coefficients of modular forms", Modular Functions of One Variable IV, Lecture Notes in Math., No. 476, Springer-Verlag, Berlin, 1975, p. 149.

105. H. P. F. Swinnerton-Dyer, On $\ell$-adic representations and congruences for coefficients of modular forms (II), Modular Functions of One Variable V, Lecture Notes in Math., No. 601, Springer-Verlag, Berlin, 1977, pp. 63-90.

106. H. P. F. Swinnerton-Dyer, Congruence properties of $\tau(n)$, Ramanujan Revisited (G. E. Andrews, R. A. Askey, B. C. Berndt, K. G. Ramanathan, and R. A. Rankin, eds.), Academic Press, Boston, 1988, pp. 289-311.

107. G. N. Watson, Über Ramanujansche Kongruenzeigenschaften der Zerfällungsanzahlen, I, Math. Z. 39 (1935), 712-731.

108. G. N. Watson, Ramanujans Vermutung über Zerfällungsanzahlen, J. Reine Angew. Math. 179 (1938), 97-128. 
109. G. N. Watson, A table of Ramanujan's function $\tau(n)$, Proc. London Math. Soc. 51 (1948), $1-13$.

110. J. R. Wilton, On Ramanujan's arithmetical function $\sigma_{r, s}(n)$, Proc. Cambridge Philos. Soc. 25 (1929), 255-264.

111. J. R. Wilton, Congruence properties of Ramanujan's function $\tau(n)$, Proc. London Math. Soc. 31 (1930), 1-10.

112. H. S. Zuckerman, Identities analogous to Ramanujan's identities involving the partition function, Duke Math. J. 5 (1939), 88-110.

Department of Mathematics, University of Illinois, 1409 West Green St., Urbana, IL 61801, USA

E-mail address: berndt@math.uiuc.edu

Department of Mathematics, Pennsylvania State University, State College, PA 16802, USA

E-mail address: ono@math.psu.edu 